\title{
Conductance of one-dimensional quantum wires
}

\author{
K.-I. Imura, K.-V. Pham, P. Lederer and F. Piéchon \\ Laboratoire de Physique des Solides, Bât. 510, CNRS, Université Paris-Sud, 91405 Orsay, France
}

(November 2, 2018)

\begin{abstract}
We discuss the conductance of quantum wires (QW) in terms of the Tomonaga-Luttinger liquid (TLL) theory. We use explicitly the charge fractionalization scheme which results from the chiral symmetry of the model. We suggest that results of the standard two-terminal (2T) conductance measurement depend on the coupling of TLL with the reservoirs and can be interpreted as different boundary conditions at the interfaces. We propose a three-terminal (3T) geometry in which the third contact is connected weakly to the bulk of TLL subjected to a large bias current. We develop a renormalization group (RG) analysis for this problem by taking explicitly into account the splitting of the injected electronic charge into two chiral irrational charges. We study in the presence of bulk contact the leading order corrections to the conductance for two different boundary conditions, which reproduce in the absence of bulk contact, respectively, the standard $2 \mathrm{~T}$ source-drain (SD) conductance $G_{\mathrm{SD}}^{(2)}=e^{2} / h$ and $G_{\mathrm{SD}}^{(2)}=g e^{2} / h$, where $g$ is the TLL charge interaction parameter. We find that under these two boundary conditions for the end contacts the $3 \mathrm{~T}$ SD conductance $G_{\mathrm{SD}}^{(3)}$ shows an UV-relevant deviation from the above two values, suggesting new fixed points in the ohmic limit. Non-trivial scaling exponents are predicted as a result of electron fractionalization.
\end{abstract}

\section{INTRODUCTION}

Interacting electrons in one spatial dimension (1D) are one of the best examples of strongly correlated fermionic systems. They are usually discussed in terms of the Tomonaga-Luttinger liquid (hereafter TLL). The latter has allowed to discuss in a precise fashion the breakdown of the Fermi liquid picture which is a good description of interacting electrons in broad band metallic three dimensional systems. In $1 \mathrm{D}$, there are no quasi-particles corresponding to a free electron with charge $-e$ and spin $1 / 2$ : the electron Green's function exhibits no quasi-particle pole, the density of states at the Fermi level vanishes at the Fermi level, and behaves as a power law with non integer exponents as a function of energy; last but not least, spin degrees of freedom are dynamically split from charge degrees of freedom. Both propagate at different velocities.

The TLL is usually understood in terms of the density fluctuations at finite wave vector, and zero wave vector "zero modes". Recently, however, taking advantage of the chiral symmetry, a new approach of the TLL Hamiltonian succeeded in formulating its physics in terms of generally irrational excitations, i.e., excitations which may have dynamically independent irrational charges or spin [1]. This constitutes a generalization of the Laughlin fractional charge excitations which have been observed in fractional quantum Hall (FQH) samples [2,3]. More precisely, the irrational excitations have been shown to be eigenstates of the TLL hamiltonian in the chiral representation. Their wave functions are formally isomorphic to Laughlin wave functions for $\mathrm{FQH}$ states. We consider hereafter a spinless TLL for simplicity. The irrational charges carried by the irrational excitations are created in chiral pairs with one excitation moving to the left and the other to the right; the charges carried by each possible pair of states form a 2D manyfold $\left(Q_{+}, Q_{-}\right)$, where $Q_{ \pm}=\frac{1}{2}(N \pm g J)$ with $N$ and $J$ being integers having the same parity: $(-1)^{N}=(-1)^{J}$. $N$ and $J$ are standard zero-mode quantum numbers associated, respectively, with the total charge and the persistent current of the system. [4] $g$ is the so-called TLL parameter, which contains all the relevant information of the electron-electron interaction. $g$ takes the value, $0<g<1$ for a standard repulsive interaction, whereas $g=1$ for non-interacting 1D fermions.

The main question to be asked is whether or not these irrational charge excitations are observable. Consider an electron incident in the middle of TLL either of infinite length or sufficiently far away from the boundaries so that the chiral symmetry be preserved. The injected electron splits into two eigen excitations which have irrational charges $Q_{+}=(1+g) / 2, Q_{-}=(1-g) / 2$ (or vice versa), and propagate in opposite directions. This picture is quite reminiscent of a three-terminal conductance measurement in which the third terminal is attached to the middle of QW connected to the source $(\mathrm{S})$ and drain (D) (See Fig. 1). The main findings of this paper are that the standard conductance measurement done in this geometry does provide some information on the charge fractionalization. More precisely, we consider a source-drain (SD) conductance $G_{\mathrm{SD}}^{(3)}$, under a large bias current $I_{\text {bias }}$ circulating through the QW between $\mathrm{S}$ and $\mathrm{D}$, and in the presence of bulk-injected current $i_{\text {bulk }}$. The third terminal, or a bulk contact, at voltage $V$ is then connected by an ohmic wire either to the $\mathrm{S}$ or to the $\mathrm{D}$. We consider the case of small $i_{\text {bulk }}$, i.e., the case in which $-e V$ is close to the chemical potential of the reservoir from which $i_{\mathrm{bulk}}$ is 
provided. We find under these circumstances $G_{\mathrm{SD}}^{(3)}$ is subjected to a change characterized by unusual scaling exponents which take different values depending on $G_{\mathrm{SD}}^{(2)}$ in the absence of $i_{\text {bulk. }}$. In Sec. III we will explain in length that different values of $G_{\mathrm{SD}}^{(2)}$ can be interpreted as different boundary conditions at the end contacts to $\mathrm{S}$ and D. In this paper, we highlight two specific boundary conditions which correspond to $G_{\mathrm{SD}}^{(2)}=e^{2} / h$ ( $\leftrightarrow$ boundary condition A) and $G_{\mathrm{SD}}^{(2)}=g e^{2} / h(\leftrightarrow$ boundary condition B). In these terms we found a non-trivial scaling exponent $(2 \Delta)^{2}-1$ (See Eq. (28)) under a specific boundary condition A, whereas a standard scaling exponent $2 \Delta-1$ (See Eq. (29)) for boundary condition B, which is simply related to the anomalous scaling dimension $\Delta$ of the TLL electron operator. This result is a remarkable consequence of electron fractionalization under a stationary bias current $I_{\text {bias }}$.

A motivation of this paper is therefore closely related to the so-called "conductance puzzle" of the QW: an apparent contradiction among different theoretical and experimental results for the two-terminal $(2 \mathrm{~T})$ conductance $G_{\mathrm{SD}}^{(2)}$ in the ohmic regime. [5] In spite of the theoretical prediction that the interaction should renormalize the conductance as $G_{\mathrm{SD}}^{(2)}=g e^{2} / h$, [8] one of the first conductance measurements on a QW by Tarucha et al. [9] has found a non-renormalized universal conductance $G_{\mathrm{SD}}^{(2)}=e^{2} / h$ for an interacting system. On the contrary, in the case of the fractional quantum Hall $(\mathrm{FQH})$ edge mode, an example of a chiral TLL [10], the Hall conductance, usually measured in a four-terminal geometry, is maximally renormalized at a topological number: $G_{\mathrm{H}}=\nu e^{2} / h[11$.

It turns out that a rapidly growing number of experimental results is now available, on the conductance of QW 12 14 and of carbon nanotubes 15 17]. Carbon nanotubes 18, 19 have been expected since their discovery [20] to be ideal 1D quantum wires. The single-walled nanotubes (SWNT) have four conducting channels indicating an expected quantized conductance: $G_{\mathrm{SD}}=4 e^{2} / h$ [21]. The ballistic transport in carbon nanotubes was first observed in only one channel of multi-walled nanotubes (MWNT) with $G_{\mathrm{SD}} \sim 2 e^{2} / h[22$. The temperature and bias voltage dependence of the conductance reported in Ref. [15] do suggest that this system is a strongly correlated $1 \mathrm{D}$ electronic liquid. The TLL theory for carbon nanotubes [23] has suggested an interaction parameter $g$ in the range $0.2-0.3$.

Recent data on the conductance of QW and carbon nanotubes display a variety of results. In Ref. [12,13], significant deviations from the quantized value $e^{2} / h$ were observed. In Ref. [16], the observed conductance exhibits fluctuations versus Fermi energy approaching the unrenormalized theoretical value $4 e^{2} / h$ as the temperature is lowered. On the other hand, in the experiment by Kasumov et al. 17] the isolated SWNT exhibits a resistance which saturates at low temperature (in the pres- ence of a sufficiently intense magnetic field) to a factor of about 0.25 times the expected unrenormalized value. In our route to suggesting experimental ways of observing irrational excitations, we had to spend some time trying to understand this variety of results. Our understanding, as explained in the body of this paper, is that two terminal conductance measurements should indeed display this variety of results, which may be understood as expressing a variety of boundary conditions at the end contacts.

The main effort of this paper is devoted to studying more involved experimental geometries than two terminal ones. To our knowledge the only way to describe injection of electrons in a QW through a weak bulk contact is to resort to the irrational excitation picture, which should be taken into account in the theoretical description of this process. Thus, examining electron injection, from one (various) weak bulk contact(s), in a QW connected to reservoirs at its ends, should lead to specific experimental predictions, as we argue in the body of this work. We were stimulated in that direction by the work of Chamon and Fradkin [25]. That work deals with the FQH effect and examines the conductance of a FQH bar (See Sec. III-C). In the case of the non-chiral liquid, one cannot easily manufacture electrical contacts which inject electrons only in one chiral eigen-mode of the TLL Hamiltonian density, so that one cannot use the results of [25]. One must actually solve the problem of the nonchiral TLL with many leads.

We derive new scaling exponents associated with the currents injected from bulk contacts. More generally we discuss effects which are derived using the irrational excitation picture, allowing measurements of $g$ through multi-terminal conductance measurements. Deviations from the unrenormalized perfect conductance value are predicted in the ohmic limit. In the renormalization group (RG) picture this naturally suggests a possibility of new intermediate fixed points. We have not been able, though, to prove that our results are unique predictions of the irrational excitation scheme, so that experimental observation of, say, the new scaling exponent mentionned above would be at best a plausibility argument in favour of this scheme. The discussion of shot-noise experiments is also left for a future publication. 26]

This paper is organized as follows: Sec. II describes the model we are studying. That section is an attempt to clarify the notion of the chemical potential for (interacting) eigen-modes, as opposed to the chemical potential for bare electrons. Sec. III discusses equilibration of 1D conductors with the 3D reservoirs: in actual experiments, which are the particles which equilibrate with the reservoirs: the bare electrons, or the eigen-modes of the TLL ? This analysis allows a physical interpretation of our boundary conditions. In Sec. IV we solve our chiral electric circuit equations using the technology developed in Sec. III. Sec. V is devoted to the RG analysis of a bulk contact. Using our fractional scaling analysis, we demonstrate that a non-trivial scaling exponent appears 
as a result of a specific boundary condition (boundary condition A). Sec. VI is a generalization to many bulk contacts. Some remarks on the application to SWNT and MWNT will be found in Sec. VII. Our conclusions are discussed in Sec. VIII.

\section{MODEL, NOTATIONS AND CHIRAL DENSITIES}

Of importance to us in this paper is the distinction to be made between bare chiral electron densities and eigen-modes chiral densities of the TLL. The bare chiral electron densities $\rho_{ \pm}^{(0)}(x, t)$ correspond to the densities of electrons created either at the left or right Fermi points of a non-interacting system. The total electronic density $\rho(x, t)$ and the current density $j(x, t)$ are related to $\rho_{ \pm}^{(0)}(x, t)$ as $\rho(x, t)=\rho_{+}^{(0)}+\rho_{-}^{(0)}$, $j(x, t)=v_{F}\left(\rho_{+}^{(0)}-\rho_{-}^{(0)}\right)$. In the non-interacting system the bare chiral electron densities are indeed two independent eigenmodes of the system: $\rho_{ \pm}^{(0)}(x, t)=\rho_{ \pm}^{(0)}\left(x \mp v_{F} t\right)$. It is however no longer true in the TLL. In the interacting system the left and right-moving electrons of the noninteracting system are strongly coupled together; accordingly, the bare electronic densities are no longer chiral. In order to clarify this point we consider the harmonic hamiltonian density of the spinless TLL,

$$
\mathcal{H}_{\mathrm{TLL}}=\frac{u}{2}\left[\frac{1}{g}\left(\frac{\partial \Phi(x, t)}{\partial x}\right)^{2}+g \Pi(x, t)^{2}\right],
$$

where we have introduced the standard phase field $\Phi$ related to the electron density by: $\rho(x, t)=\frac{1}{\sqrt{\pi}} \frac{\partial \Phi(x, t)}{\partial x}$, and its conjugate canonical momentum $\Pi(x, t)$. $u=v_{F} / g$ is the dressed velocity. Note also that the continuity equation shows that the current density is simply: $j(x, t)=-\frac{1}{\sqrt{\pi}} \frac{\partial \Phi(x, t)}{\partial t}$. The stationary components of $\rho(x, t)$ and $j(x, t)$ are the zero modes: $N=\int_{-L / 2}^{L / 2} \rho(x, t)$, $J=\int_{-L / 2}^{L / 2} j(x, t)$, which obey the fermionic selection rule $(-1)^{N}=(-1)^{J}$ 朋. Using Hamilton equations: $u g \Pi(x, t)=\frac{\partial \Phi(x, t)}{\partial t}, \frac{u}{g} \frac{\partial^{2} \Phi(x, t)}{\partial x^{2}}=\frac{\partial \Pi(x, t)}{\partial t}$, one finds immediately

$$
\left(\frac{\partial}{\partial x} \mp \frac{1}{u} \frac{\partial}{\partial t}\right)\left[\frac{\partial \Phi(x, t)}{\partial x} \pm g \Pi(x, t)\right]=0 .
$$

This shows that $\rho_{ \pm}(x, t)=\frac{1}{2 \sqrt{\pi}}\left[\frac{\partial \Phi(x, t)}{\partial x} \pm g \Pi(x, t)\right]$ are indeed chiral eigenmodes of the system: $\rho_{ \pm}(x, t)=$ $\rho_{ \pm}(x \mp u t)$. Observing that $\rho(x, t)=\rho_{+}+\rho_{-}, j(x, t)=$ $u\left(\rho_{+}-\rho_{-}\right)$, one concludes that $\rho_{+}$and $\rho_{-}$correspond to a different decomposition of the total density into chiral densities from the non-interacting case. These eigenmode chiral densities mix both left and right moving electrons, since the bare chiral densities (obtained when $g=1)$ are: $\rho_{ \pm}^{(0)}=\frac{1}{2 \sqrt{\pi}}\left[\frac{\partial \Phi(x, t)}{\partial x} \pm \Pi(x, t)\right]$. In terms of these eigenmode chiral densities obeying the anomalous Kac-Moody commutation relations, the hamiltonian density splits into two commuting chiral parts: $\mathcal{H}=\frac{\pi u}{g} \rho_{+}^{2}+\frac{\pi u}{g} \rho_{-}^{2}=\mathcal{H}_{+}+\mathcal{H}_{-}$. The stationary component of $\rho_{ \pm}(x, t)$ are nothing but the chiral charges $Q_{ \pm}=\int_{-L / 2}^{L / 2} \rho_{ \pm}(x, t)$ 27]. It is convenient to introduce a vector notation for the chiral densities. The dressed eigenmode density $\vec{\rho}=\left[\begin{array}{l}\rho_{+} \\ \rho_{-}\end{array}\right]$is related to the bare density $\vec{\rho}^{(0)}=\left[\begin{array}{l}\rho_{+}^{(0)} \\ \rho_{-}^{(0)}\end{array}\right]$ in the matrix equation as $\vec{\rho}=\boldsymbol{\Omega} \vec{\rho}^{(0)}$, where the matrix $\boldsymbol{\Omega}=\frac{1}{2}\left[\begin{array}{cc}1+g & 1-g \\ 1-g & 1+g\end{array}\right]$ characterizes the fractionalization of electronic charge $-e$.

In the absence of applied external voltage $V_{S}-V_{D}$ the average current $I=\langle j(x, t)\rangle$ is zero. In order to drive a net current through the sample, let us allow for independent variations of the left and right bare chemical potentials. The possibility to adjust them independently expresses the chiral separation of TLL. This is accomplished by adding a chemical potential to the hamiltonian. But once again a distinction should be made between bare chemical potentials $\vec{\mu}^{(0)}=\left[\begin{array}{c}\mu_{+}^{(0)} \\ \mu_{-}^{(0)}\end{array}\right]$ corresponding to a variation of the bare electron densities and eigenmode chemical potentials $\vec{\mu}=\left[\begin{array}{l}\mu_{+} \\ \mu_{-}\end{array}\right]$corresponding to the eigen-mode chiral densities. More precisely, $\vec{\mu}^{(0)}$ and $\vec{\mu}$ are defined, respectively, by minimizing

$$
\begin{aligned}
\mathcal{H}_{\mathrm{TLL}}-\mu_{+}^{(0)} \rho_{+}^{(0)}-\mu_{-}^{(0)} \rho_{-}^{(0)} & =\frac{u \pi}{2 g} \vec{\rho}^{(0) T} \boldsymbol{\Omega}^{2} \vec{\rho}^{(0)}-\vec{\mu}^{(0) T} \vec{\rho}^{(0)} \\
\mathcal{H}_{\mathrm{TLL}}-\mu_{+} \rho_{+}-\mu_{-} \rho_{-} & =\frac{u \pi}{2 g} \vec{\rho}^{T} \vec{\rho}-\vec{\mu}^{T} \vec{\rho}
\end{aligned}
$$

Completing the square densities, one finds $\vec{\mu}^{(0)}=$ $\frac{u \pi}{g} \Omega^{2}\left\langle\vec{\rho}^{(0)}\right\rangle, \vec{\mu}=\frac{u \pi}{g}\langle\vec{\rho}\rangle=\frac{u \pi}{g} \Omega\left\langle\vec{\rho}^{(0)}\right\rangle$ [5,28]. Comparing the two expressions, the relation between bare and dressed chemical potential is found to be $\vec{\mu}^{(0)}=\Omega \vec{\mu}$. Note that $\vec{\mu}^{(0)}$ and $\vec{\mu}$ obey the same linear transformation as the one for $\vec{\rho}^{(0)}$ and $\vec{\rho}$ except that the roles of bare elctrons and of the chiral eigenmodes are exchanged.

It would be worth mentioning here that in the fourterminal conductance measurement by Picciotto et. al. the resistance data (Fig. 3 of [14]) shows that the chemical potential $\mu$ coupled to additional probes (probe A and $\mathrm{B})$ is neither bare nor dressed chiral chemical potentials. Instead the additional probes seem to be coupled almost equally to both chiralities; $\mu$ is coupled to the total density: we should rather minimize the hamiltonian density $\mathcal{H}_{\mathrm{TLL}}-\mu \rho$ to find $\mu=\frac{\mu_{+}+\mu_{-}}{2}$. If the electronic transport through the conductor is perfectly balistic, this chemical potential $\mu$ is uniform throughout the conductor, which explains the data of Picciotto et. al. 
In the presence of an electric field the chemical potential becomes an electrochemical potential and one may introduce the following chiral voltages: $\vec{\mu}^{(0)}=-e \vec{V}^{(0)}=$ $\left[\begin{array}{l}-e V_{+}^{(0)} \\ -e V_{-}^{(0)}\end{array}\right], \vec{\mu}=-e \vec{V}=\left[\begin{array}{l}-e V_{+} \\ -e V_{-}\end{array}\right]$. They are related, of course, via the relation $\vec{V}=\Omega \vec{V}^{(0)}$. Thus the total current $I=-e\langle j(x, t)\rangle$ can be expressed either in terms of the bare voltages $I=-e u[1,-1] \vec{\rho}=\frac{e^{2}}{h}[1,-1] \vec{V}^{(0)}$ or in terms of the dressed eigenmode voltages as $I=$ $-e v_{F}[1,-1] \vec{\rho}^{(0)}=g \frac{e^{2}}{h}[1,-1] \vec{V}$, where we have used $[1,-1] \boldsymbol{\Omega}=g[1,-1]$. Note that we are working in the unit where $\hbar=1$. These relations together with $\vec{V}=\Omega \vec{V}^{(0)}$ play a central role in later sections when the sample is connected to the reservoirs through various boundary conditions.

We have summarized the bosonized formulation of TLL as well as its response to external electric field by emphasizing the difference between bare and eigen-mode chiral densities. Let us now turn to a discussion of its implications on the transport through TLL.

\section{BOUNDARY CONDITIONS AT THE END CONTACTS - SCREENING AND EQUILIBRATION}

In the approach developped by Landauer and Büttiker for non-interacting electrons, the chiral chemical potentials $-e V_{+},-e V_{-}$of the bulk sample are equilibrated with that of the reservoir from which the electrons are injected [24]: $V_{S}=V_{+}, V_{D}=V_{-}$. Whereas in the bulk the total current $I$ is related to $V_{+}, V_{-}$as $I=\frac{e^{2}}{h}\left(V_{+}-V_{-}\right)$. Thus the above boundary condition ensures the 2T SD conductance, usually defined as $G_{\mathrm{SD}}^{(2)}=I /\left(V_{S}-V_{D}\right)$, to be given by the standard unit conductance: $G_{\mathrm{SD}}^{(2)}=e^{2} / h$.

Let us now switch on the interaction. As we have seen in the last section, the conductance of the system as measured against either the bare or eigen-mode voltage yields therefore different values, e.g., if the conductance is measured against $V_{+}-V_{-}$, this gives the conductance $g e^{2} / h$ in the bulk, which is reminiscent of the four-terminal measurement in FQH bar [1]. In the case of the nonchiral liquid, a four-terminal measurement analogous to the one in Ref. [1] is difficult to realize, since one cannot easily manufacture electrical contacts which are coupled only to one of the chiral eigenmodes of TLL Hamiltonian. In the experiment by Picciotto et. al. the resistance data (Fig. 3 of [14]) shows that the voltage probes (probe A and B) are coupled almost equally to both chiralities. On the other hand, if the conductance is measured, under certain circumstances, against $V_{+}^{(0)}-V_{-}^{(0)}$, then it gives $e^{2} / h$. We believe that the value taken by the conductance, when the bulk sample is connected to the current reservoirs, is, a matter of coupling between the sample and the reservoirs. In the followoing we will formulate this in a more systematic way, i.e., in the form of boundary conditions at the end contacts.

\section{A. Equilibration with bare electrons - Screening by a metallic gate}

Let us first consider the boundary condition discussed in refs. 5 5, 30, 29, 31. This boundary condition has been labelled a "radiative" boundary condition in Ref. [31]. With this boundary condition the particles emitted by the left reservoir are then in equilibrium with the bare electrons:

$$
\left\{\begin{array}{l}
V_{S}=V_{+}^{(0)}=\left[\begin{array}{ll}
1 & 0
\end{array}\right] \boldsymbol{\Omega} \vec{V} \\
V_{D}=V_{-}^{(0)}=\left[\begin{array}{ll}
0 & 1
\end{array}\right] \boldsymbol{\Omega} \vec{V}
\end{array},\right.
$$

where the matrix $\boldsymbol{\Omega}$ has been defined as

$$
\boldsymbol{\Omega}=\frac{1}{2}\left[\begin{array}{ll}
1+g & 1-g \\
1-g & 1+g
\end{array}\right]
$$

Let us first recall that the conductance defined with this boundary condition is indeed $e^{2} / h$ independently of $g$. Recall the relation between bare and dressed voltages: $\vec{V}=\Omega \vec{V}^{(0)}$. The bias voltage $V_{S}-V_{D}=[1,-1] \vec{V}^{(0)}$ can be written as $V_{S}-V_{D}=[1,-1] \Omega \vec{V}=g[1,-1] \vec{V}$, where we have used $[1,-1] \boldsymbol{\Omega}=g[1,-1]$. The total current $I$ can be expressed either in terms of the bare bias voltages $I=\frac{e^{2}}{h}[1,-1] \vec{V}^{(0)}$ or in terms of the dressed eigenmode voltages as $I=g \frac{e^{2}}{h}[1,-1] \vec{V}$. It then follows that the conductance is given by $G_{\mathrm{SD}}^{(2)}=e^{2} / h$ independently of $g$.

Another important remark is that this boundary condition requires the existence of a metallic gate along the $1 \mathrm{D}$ sample 30,31]. When a certain amount of charge $Q$ is injected from the reservoir through an end contact, the TLL system cannot screen this charge completetely because of this boundary condition, instead $Q_{\mathrm{TLL}}=-\left(1-g^{2}\right) Q$ is induced in TLL. In order for the charge conservation $Q+Q_{\mathrm{TLL}}+Q_{\text {gate }}=0$ to be satisfied there needs to exist a metallic gate providing for a screening charge $Q_{\text {gate }}=-g^{2} Q$ [30,31. The existence of metallic gate also explains a short-range interaction in 1D quantum wire which ensures a finite parameter $g$ of TLL model. In the experiment by Tarucha et. al. [9] the $1 \mathrm{D}$ sample was indeed screened by the metallic gate.

\section{B. Equilibration with dressed eigenmodes - No screening gate}

Knowing that the boundary condition (3) requires the existence of metallic gate one naturally asks the question what will be the corresponding boundary conditions in the absence of screening by a metallic gate. TLL with finite $g$ (long-range interaction cut off by the finite length 
of the sample and the width of the tube) without screening gate is indeed realized in a SWNT [21]. The boundary condition which ensures $Q+Q_{\mathrm{TLL}}=0$ with no reference to the metallic gate is, in fact, $V_{+}=V_{S}, V_{-}=V_{D}$. The dressed eigenmode voltages are equilibrated with those of the reservoirs, i.e., the particles emitted from the left (right) reservoir are moving to the right (left) and are in equilibrium with the right (left)-moving eigenstates of the TLL. If this naive picture is indeed the case, the conductance in terms of the voltage difference between the reservoirs, is obtained immediately from the relation $I=g \frac{e^{2}}{h}[1,-1] \vec{V}$, i.e., $G_{\mathrm{SD}}^{(2)}=g e^{2} / h$. The conductance is fully renormalized [26].

\section{Intermediate possibilities}

Are there intermediate possibilities, i.e., situations where neither bare electrons nor eigenstates are in equilibrium with the reservoir? In the case of FQH edge mode, i.e., if one replaces in the above discussion the 1D TLL sample by a FQH bar at bulk filling factor $\nu$, it was shown in Ref. 25 that depending on the number $N_{L}$ $\left(N_{R}\right)$ of left (right) strong point like contacts with the left (right) reservoir the $S D$ conductance of a $F Q H$ bar $G_{\mathrm{SD}}$ varies from $G_{\mathrm{SD}}=e^{2} / h$ to $G_{\mathrm{SD}}=\nu e^{2} / h$. The first case (boundary condition A) in which the $2 \mathrm{~T}$ conductance is not fractional but integral $\left(G_{\mathrm{SD}}^{(2)}=e^{2} / h\right)$ corresponds to a single tunneling point contact: $N_{L}=N_{R}=1$. The second case (boundary condition B) where the end contacts are in equilibrium with dressed eigenmodes corresponds to an infinite number of tunneling point contacts between each reservoir and the sample: $\left(N_{L}, N_{R}\right) \rightarrow(\infty, \infty)$. The third possibility (boundary condition $\mathrm{C}$ ) arises in between, when there is a finite number of tunneling quantum point contacts.

Turning to the case of the quantum wire, which of these three cases is relevant experimentally? A first model is that of the inhomogeneous TLL, in which the reservoirs are modelized as TLL at $g=1$ (1D free fermions). [5] We observe that this model implicitly describes a transmission of the current through a single point contact. Furthermore it implies the presence of a screening gate. This confirms that a single contact between the reservoirs is not enough for equilibration of the $1 \mathrm{D}$ sample with the reservoirs as we surmised, yielding therefore an unrenormalized conductance. A second series of explanations rely on the assumption that the conductance measured is the ratio $I / V_{\text {loc }}$ of the current to a so-called total local field, which sums a contribution from the external electrical field plus some response of the TLL, rather than the ratio of the current to the external potential as usual [34]. It is then shown by a diagrammatical analysis that the local field is $E_{\text {loc }}=E_{\text {ext }} / g$ 35. The ratio $I / V_{\text {ext }}$ being assumed as in the initial work by Kane and Fisher [32] to be equal to $g$, this yields an unrenormalized conductance $I / V_{\text {loc }}=1$. That analysis is doubtful because experi- mentally the external field is fixed which means that one really measures the ratio $I / V_{\text {ext }}$. By contrast, in our approach what is measured is indeed the ratio $I / V_{\text {ext }}=1$, although we agree on the relation $E_{\text {loc }}=E_{\text {ext }} / g$. Note that this follows from elementary considerations on chiral modes (see section II).

We stress that although the boundary condition A seems to be realized in the experiments when a screening gate is present, this does not bar the possibility that, in different set-ups, boundary condition $\mathrm{B}$ or $\mathrm{C}$ might apply. In the experiment by Kasumov et. al. [17] the isolated SWNT sample $S T_{1}$ exhibits a resistance $R$ which saturates at high temperatures to $R \sim 25 \mathrm{k} \Omega$ (see Fig. $2 \mathrm{~B}$ of Ref. [17]). This corresponds to the conductance $G_{\mathrm{SD}}^{(2)} \sim e^{2} / h$, which is smaller by a factor $1 / 4$ than $G_{\mathrm{SD}}^{(2)}=4 e^{2} / h$ expected from the boundary condition (3). Note that this experiment was done in the absence of screening gate, where the boundary condition B might apply. Since $g$ is typically in the range 0.2 to 0.3 , the observed conductance $G_{\mathrm{SD}}^{(2)} \sim e^{2} / h$ is indeed reminiscent of the renormarized conductance: $G_{\mathrm{SD}}^{(2)}=4 g e^{2} / h$.

The inhomogeneous TLL model [5] assumes by construction an injection of current through a single end contact for each reservoir. But the current might be injected through bulk contacts in addition to being injected through end contacts. In further sections of this paper we address the issue of bulk contacts using the perpurbative $\mathrm{RG}$ analysis for bulk tunneling.

\section{ONE BULK CONTACT IN THE PRESENCE OF A LARGE BIAS CURRENT}

In the following we will consider a model where the sample is connected to the reservoir not only through the end contacts but also through some bulk contacts. For the end contact, depending on the presence or absence of screening gate, the boundary conditions (A) or (B) will be applied. We will mainly focus on the case where the two end contacts impose a large stationary current $I_{\text {bias }}$ in the quantum wire. In this case the additional current injected through the bulk contact can be treated as a small perturbation. We will develop later a RG analysis which is compatible with fractionalization scheme, which will tell us the scaling behavior of this bulk injected current. In order to utilize the results of this fractional RG analysis, we first have to derive and solve the equations which describe our chiral electric circuits. We will see that the existence of a large stationary current as well as different boundary conditions play an important role in finding a new exponent in the conductance formula.

A motivation of our work is based on the findings of Ref. [1]. Following the latter work, an electron injected from a normal lead into a TLL splits into two eigen excitations which are fractional (in fact, for most repulsive interaction strengths, the correct term would be irrational). Namely, the two chiral eigen excitations have 
charges $(1+g) / 2,(1-g) / 2$, and they propagate in opposite directions with the corresponding chiral mode velocities.

Various authors have discussed the observability of those irrational excitations ge [1, 32, 36 39]. The obvious suggestion is based on electric current noise measurements [1]. However the results of Ref. 25] suggest that the parameter $g$ might also be measured in a conductance experiment with many contact points, and/or possibly different bias voltages. This is what we proceed to do below, in various situations.

Let us first consider a single bulk contact in the middle of the sample. The bulk contact has the following two effects (see Fig. 1).

1. Tunneling of Laughlin quasiparticles [1] between the two chiral modes. This is due to the backward scattering between $+k_{\mathrm{F}}$ and $-k_{\mathrm{F}}$ electrons. The total charge is conserved in this process $(N=$ $0, J= \pm 2$ ). When a macroscopic number of electrons are involved in this process, a current $i_{\text {back }}$ is back-scattered from (+)-chirality to (-)-chirality.

2. Tunneling of electrons into or out of the bulk sample through the bulk contact, i.e., injection or ejection of electrons throught the bulk contact. The total charge is increased or decreased by 1: $N=$ $\pm 1, J= \pm 1$, respectively, for the tunneling of $+k_{\mathrm{F}}\left(-k_{\mathrm{F}}\right)$ electrons. When a current $i_{\text {bulk }}$ is injected through the bulk contact into the sample, it splits into two parts $i_{+}$and $i_{-}$corresponding, respectively, to an eigen modes with $+(-)$-chirality: $i_{\text {bulk }}=i_{+}+i_{-}$.

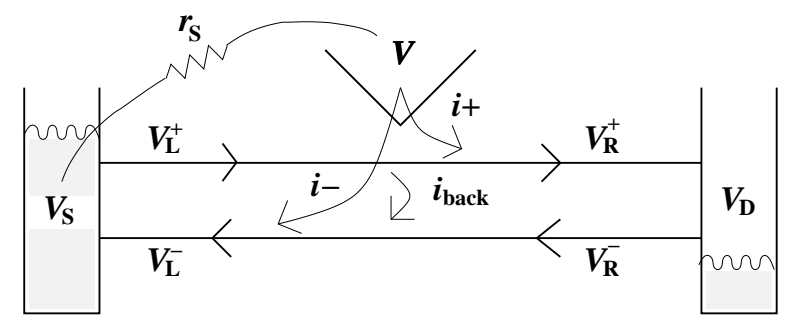

FIG. 1. A bulk contact in the presence of a large stationary current $I_{\text {bias. }}$. The chiral symmetry of the TLL is not destroyed by the bulk contact. - At the bulk contact a current $i_{\text {back }}$ is back-scattered from (+)-chirality to (-)-chirality due to the tunneling of Laughlin quasiparticle. A net current $i_{\text {bulk }}$ is injected through the bulk contact into the sample. As soon as it is injected into the TLL sample, it splits into two parts $i_{+}$and $i_{-}$corresponding, respectively, to one of the TLL eigenmodes with $+(-)$-chirality.

In order to see how the bulk contacts influence the twoterminal conductance of the system, we develop below the voltage drop equations, i.e., a set of equations which determine the chemical potential of the system on each side of the bulk contacts. In the presence of 1 . backscattering, 2. electron injection (ejection), the voltage drop equation reads

$$
\left\{\begin{array}{c}
i_{+}-i_{\text {back }}=g \frac{e^{2}}{h}\left(V_{R}^{+}-V_{L}^{+}\right) \\
i_{-}+i_{\text {back }}=g \frac{e^{2}}{h}\left(V_{L}^{-}-V_{R}^{-}\right)
\end{array},\right.
$$

where $V_{L}^{ \pm}$and $V_{R}^{ \pm}$are the electrostatic potentials of the eigen modes on each side of the bulk contact. Using the vector notation $\vec{i}_{\text {bulk }}=\left[\begin{array}{l}i_{+} \\ i_{-}\end{array}\right], \vec{V}_{L}=\left[\begin{array}{l}V_{L}^{+} \\ V_{L}^{-}\end{array}\right]$, $\vec{V}_{R}=\left[\begin{array}{l}V_{R}^{+} \\ V_{R}^{-}\end{array}\right]$, the voltage drop equations can be rewritten in a simpler form and can be treated in a systematic way,

$$
\vec{V}_{R}-\vec{V}_{L}=\frac{\sigma_{\mathbf{z}} \vec{i}_{\text {bulk }}}{g e^{2} / h}-\frac{i_{\text {back }}}{g e^{2} / h}\left[\begin{array}{l}
1 \\
1
\end{array}\right]
$$

where $\sigma_{\mathbf{z}}=\left[\begin{array}{rr}1 & 0 \\ 0 & -1\end{array}\right]$. For later convenience we introduce the following decomposition of $\vec{i}_{\text {bulk }}$ :

$$
\vec{i}_{\text {bulk }}=i_{+k_{\mathrm{F}}} \boldsymbol{\Omega}\left[\begin{array}{l}
1 \\
0
\end{array}\right]+i_{-k_{\mathrm{F}}} \boldsymbol{\Omega}\left[\begin{array}{l}
0 \\
1
\end{array}\right],
$$

where the matrix $\boldsymbol{\Omega}$ was defined in Eq. (价. $i_{+k_{\mathrm{F}}}$ and $i_{-k_{\mathrm{F}}}$ are, respectively, the currents injected from the bulk contact through $\Psi_{+k_{\mathrm{F}}} \sim e^{i \sqrt{\pi}\left[\begin{array}{ll}1 & 0\end{array}\right] \Omega\left[\begin{array}{c}\theta_{+} \\ \theta_{-}\end{array}\right]}$, or through $\Psi_{-k_{\mathrm{F}}} \sim e^{i \sqrt{\pi}\left[\begin{array}{ll}0 & 1\end{array}\right] \Omega\left[\begin{array}{c}\theta_{+} \\ \theta_{-}\end{array}\right]}$, where $\theta_{ \pm}=\theta \mp \frac{\phi}{g}$ defined in terms of $\phi=\Phi(x=0), \theta=\Theta(x=0)$ are the values of chiral fields at the bulk contact $(x=0)$. Noticing that $[1,1] \boldsymbol{\Omega}=[1,1]$, one can easily check that the total bulk injected (ejected) current is indeed $i_{\text {bulk }}=[1,1] \vec{i}_{\text {bulk }}=i_{+k_{\mathrm{F}}}+i_{-k_{\mathrm{F}}}$.

The total current $I_{L}, I_{R}$ on each side of the bulk contact is related to $\vec{V}_{L}, \vec{V}_{R}$ via the equations: $I_{L}=$ $\frac{e^{2}}{h}[1,-1] \Omega \vec{V}_{L}=g \frac{e^{2}}{h}[1,-1] \vec{V}_{L}, I_{R}=\frac{e^{2}}{h}[1,-1] \Omega \vec{V}_{R}=$ $g \frac{e^{2}}{h}[1,-1] \vec{V}_{R}$. Let us first focus on

$$
I_{R}-I_{L}=g \frac{e^{2}}{h}[1,-1]\left(\frac{\sigma_{\mathbf{z}} \vec{i}_{\text {bulk }}}{g e^{2} / h}-\frac{i_{\text {back }}}{g e^{2} / h}\left[\begin{array}{l}
1 \\
1
\end{array}\right]\right)=i_{\text {bulk }}
$$

where we have used (6). The physical meaning of this simplest equation is far from uninteresting.

1. The total current is conserved on each side of the bulk contact, i.e., $i_{\text {back }}$ does not appear in $I_{L}-I_{R}$, reflecting the fact that the back-scattering conserves the total charge. 
2. Even though an electron injected from the bulk contact (normal lead) splits into two fractional excitations propagating in the opposite directions, leading to $i_{+}-i_{-}=[1,-1] \vec{i}_{\text {bulk }}=g\left(i_{+k_{\mathrm{F}}}-i_{-k_{\mathrm{F}}}\right)$ (see Eq. (7)), the total current added by $\vec{i}_{\text {bulk }}$ is $i_{\text {bulk }}$ independently of $g$.

Now we connect both ends of the sample to the reservoirs through either of the two boundary conditions discussed in section III.

\section{A. One bulk contact with end contacts in equilibrium with bare electrons}

Let us first consider the case where the sample is connected to the reservoirs via the boundary condition (3). Using the voltage drop equation (6), the boundary condition (3) at the end contacts can be written as

$$
\begin{aligned}
V_{S} & =\left[\begin{array}{ll}
1 & 0
\end{array}\right] \boldsymbol{\Omega} \vec{V}_{L} \\
& =\left[\begin{array}{ll}
1 & 0
\end{array}\right] \boldsymbol{\Omega}\left(\vec{V}_{R}-\frac{\sigma_{\mathbf{z}} \vec{i}_{\text {bulk }}}{g e^{2} / h}+\frac{i_{\text {back }}}{g e^{2} / h}\left[\begin{array}{l}
1 \\
1
\end{array}\right]\right) \\
V_{D} & =\left[\begin{array}{ll}
0 & 1
\end{array}\right] \boldsymbol{\Omega} \vec{V}_{R} \\
& =\left[\begin{array}{ll}
0 & 1
\end{array}\right] \boldsymbol{\Omega}\left(\vec{V}_{L}+\frac{\sigma_{\mathbf{z}} \vec{i}_{\text {bulk }}}{g e^{2} / h}-\frac{i_{\text {back }}}{g e^{2} / h}\left[\begin{array}{l}
1 \\
1
\end{array}\right]\right)
\end{aligned}
$$

Hence

$$
\begin{aligned}
V_{S}-V_{D} & =\vec{V}_{R}^{T} \boldsymbol{\Omega}\left[\begin{array}{r}
1 \\
-1
\end{array}\right]+\frac{i_{\mathrm{back}}}{g e^{2} / h}-\left[\begin{array}{ll}
1 & 0
\end{array}\right] \frac{\boldsymbol{\Omega} \sigma_{\mathbf{z}} \vec{i}_{\mathrm{bulk}}}{g e^{2} / h} \\
& =\vec{V}_{L}^{T} \boldsymbol{\Omega}\left[\begin{array}{r}
1 \\
-1
\end{array}\right]+\frac{i_{\mathrm{back}}}{g e^{2} / h}-\left[\begin{array}{ll}
0 & 1
\end{array}\right] \frac{\boldsymbol{\Omega} \sigma_{\mathbf{z}} \vec{i}_{\mathrm{bulk}}}{g e^{2} / h} .
\end{aligned}
$$

Let us first look at (11). Recall the decomposition (7). Noticing that $\boldsymbol{\Omega} \sigma_{\mathbf{z}} \boldsymbol{\Omega}=g \sigma_{\mathbf{z}}$ is a diagonal matrix, one can rewrite the bias voltage $V_{S}-V_{D}$ as

$$
V_{S}-V_{D}=\frac{h}{e^{2}}\left(I_{R}+\frac{1}{g} i_{\text {back }}-i_{+k_{\mathrm{F}}}\right)
$$

i.e., $i_{-k_{\mathrm{F}}} \Omega\left[\begin{array}{l}0 \\ 1\end{array}\right]$ in $\vec{i}_{\text {bulk }}$ does not contribute to (12). In order to see the consequence of (12) let us first consider the case: $i_{\text {bulk }}=0$, i.e., electrons are neither injected nor ejected through the bulk contact. In this case the total current in the presence of the backscattered current $i_{\text {back }}$ is given by $I=I_{L}=I_{R}=\frac{e^{2}}{h}\left(V_{S}-V_{D}\right)-\frac{1}{g} i_{\text {back. }}$. It is very interesting to compare it with the current in the absence of bulk contact: $I_{\max }=\frac{e^{2}}{h}\left(V_{S}-V_{D}\right)$. The result is obviously

$$
I_{\max }-I=\frac{1}{g} i_{\mathrm{back}}
$$

which is different from the naive expectation $I_{\max }-I=$ $i_{\text {back }}$, by a factor $1 / g$. In the derivation of (13) the boundary condition (9) has been treated carefully with $V_{S}$ and $V_{D}$ being fixed. Our result, Eq. (13), which differs from the analysis in Ref. 38], leads to an important remark on the shot-noise experiment in TLL. [26]

In order to see how the two-terminal conductance is affected by the bulk contact, we connect the bulk contact to the source $(\mathrm{S})$ through an ohmic resistance $r_{S}$. In this case the total current circulating in the system is $I=I_{R}=[1,-1] \Omega \vec{V}_{R}$. Therefore Eq. (12) means

$$
I=\frac{e^{2}}{h}\left(V_{S}-V_{D}\right)-\frac{1}{g} i_{\text {back }}+i_{+k_{\mathrm{F}}} .
$$

Another remark is that in the physically interesting case of small $i_{\text {bulk }}=i_{+}+i_{-}=i_{+k_{\mathrm{F}}}+i_{-k_{\mathrm{F}}}>0, V=$ $V_{S}-r_{S} i_{\text {bulk }}$ where $r_{S}$ is a resistance between the source and bulk contact. This means $V_{D} \ll V<V_{S}$.

When the bulk contact is connected to the drain (D), one should use (11). Thus the total current $I=I_{L}=$ $[1,-1] \boldsymbol{\Omega} \vec{V}_{L}$ can be written as

$$
I=\frac{e^{2}}{h}\left(V_{S}-V_{D}\right)-\frac{1}{g} i_{\mathrm{back}}-i_{-k_{\mathrm{F}}} .
$$

We will be interested in the case of small $i_{\text {bulk }}<0$, i.e., $V_{D}<V=V_{D}-r_{D} i_{\text {bulk }} \ll V_{S}$.

We will see in the next section that Eqs. (14,15) give rise to a non-trivial physical consequence, where we apply to them the results of our fractional scaling analysis. This is not only due to the specific boundary condition which we have chosen for the moment but also related to the existence of a large stationary current. In the absence of stationary current $I_{\text {bias }}$, i.e., when $V_{S}=V_{D}=V_{0}$, which actually implies $V_{+}=V_{-}=V_{+}^{(0)}=V_{-}^{(0)}=V_{0}$, the scaling behavior of $i_{ \pm k_{\mathrm{F}}}$ is trivial: $i_{+k_{\mathrm{F}}}=i_{-k_{\mathrm{F}}} \sim$ $\pm\left|V-V_{0}\right|^{2 \Delta}$, depending on the sign of $V-V_{0}$, where $V$ is the electrostatic potential of the bulk contact and $\Delta$ is the anomalous scaling dimension of a TLL electron. In the case of finite $\mathrm{SD}$ voltage $V_{S} \neq V_{D}$, where $V_{+}, V_{-}$, $V_{+}^{(0)}$ and $V_{-}^{(0)}$ are all different, we have to look into the details of fractional decomposition and find out the scaling law as a function of $I_{\text {bias }}$. This is done in Sec. V below.

\section{B. One bulk contact with end contacts in equilibrium with dressed eigenmodes}

Before turning to the RG analysis of bulk contacts, let us briefly look at the second boundary condition discussed in section III-B, i.e., the case where the dressed eigenmode voltages are equilibrated with those of the reservoirs: $V_{L}^{+}=V_{S}, V_{R}^{-}=V_{D}$. The particles emitted from the left (right) reservoir are moving to the right (left) and are in equilibrium with the right (left)-moving eigenstates of the TLL. This situation may be achieved 
in the SWNT in the absence of screening gate. The twoterminal conductance in the present case in the absence of bulk contacts is quantized at $G_{\mathrm{SD}}^{(2)}=g e^{2} / h$. In the case of one bulk contact connected to the source $(\mathrm{S})$, the voltage drop equation (6) implies instead of (14)

$$
I=g \frac{e^{2}}{h}\left(V_{S}-V_{D}\right)-i_{\mathrm{back}}+i_{+}
$$

where the total current $I$ is given as $I=I_{R}=$ $g[1,-1] \vec{V}_{R}$. When the bulk contact is connected to the drain (D), one finds instead

$$
I=g \frac{e^{2}}{h}\left(V_{S}-V_{D}\right)-i_{\text {back }}-i_{-} .
$$

Note that in Eqs. 16,17 ) the correction due to the bulk contact enters as either $i_{+}$or $i_{-}$in contrast to Eqs. $(14,15)$. As a result, we will see that the leading scaling exponents which appear in Eqs. (16,17) are different from the ones in (14 15) and that they are simply related to the anomalous scaling dimension $\Delta$ of the TLL electron operator. All these issues concerning the scaling behavior of Eqs. (14,15,16,17) will be discussed in detail in the next section. However the reader who is more interested in the physical consequences of the analysis in this section than a detailed deriviation of $\mathrm{RG}$ equations can skip to Eqs. 26,27 ) and Sec. V-C.

\section{RG ANALYSIS FOR THE 3T CONDUCTANCE MEASUREMENT}

We are mostly interested in the leading order corrections to the conductance in the presence of bulk contact under two different boundary conditions discussed in Sec. III-A and Sec. III-B. It is not difficult to realize using the standard scaling analysis that the backscattered current is IR-relevant whereas the bulk-injected current is UV-relevant for repulsive interaction $(0<g<1)$, i.e., $i_{\text {back }} /\left(V_{S}-V_{D}\right)\left(i_{\text {bulk }} /\left(V_{S}-V_{D}\right)\right)$ scales to smaller (larger) values as the SD voltage $V_{S}-V_{D}$ is increased. However as we will see later in this section, more precise understanding of the scaling will become necessary in the analysis of Eqs. (14,15,16,17). In particular it turns out less trivial in a chirally separated system but essential in the discussion of conductance to identify the cutoff energy scale $\Lambda$ which defines the upper limit of physically meaningful energy scales.

In the case of standard backscattering problem without $i_{\text {bulk }}$, 32 there are two fixed points on the RG flow diagram, usually drawn in terms of the conductance $G_{\mathrm{SD}}^{(2)}$ ( $y$-axis) as a function of $\Lambda$ (x-axis). The two fixed points are at $G_{\mathrm{SD}}^{(2)}=0(\Lambda=0)$ and either at $G_{\mathrm{SD}}^{(2)}=e^{2} / h$ or at $G_{\mathrm{SD}}^{(2)}=g e^{2} / h(\Lambda \rightarrow \infty)$. Here we are interested in the latter UV (ohmic) limit in the presence of $i_{\text {bulk. We }}$ will see that the $3 \mathrm{~T}$ SD conductance $G_{\mathrm{SD}}^{(3)}$ shows an UVrelevant deviation and interpolates between the standard $2 \mathrm{~T}$ values: $G_{\mathrm{SD}}^{(2)}=e^{2} / h$ and $G_{\mathrm{SD}}^{(2)}=g e^{2} / h$.

We start with the Euclidian Lagrangian density for non-chiral TLL,

$$
\mathcal{L}_{\mathrm{TLL}}=\frac{u}{2}\left[\frac{1}{g}\left(\frac{\partial \Phi}{\partial x}\right)^{2}+g\left(\frac{\partial \Theta}{\partial x}\right)^{2}\right]+i \frac{\partial \Phi}{\partial \tau} \frac{\partial \Theta}{\partial x}
$$

We do not consider for the time being the effects of end contacts. On the other hand we take into account the existence of a stationary current $I_{\text {bias }}$. In the absence of bulk contact, the TLL has two chiral eigen modes respectively at voltages $V_{+}$and $V_{-}$. These voltages $V_{+}$ and $V_{-}$are related to the stationary current $I_{\text {bias }}$ via $I=g \frac{e^{2}}{h}[1,-1] \vec{V}$.

The bulk contact is at $x=0$. All the bosonic fields involved in backscattering or tunneling should be understood as those at $x=0$. Therefore it is convenient to integrate out the continuum degrees of freedom in (18) to obtain the effective action at $x=0$.

$$
\begin{aligned}
S_{0} & =\frac{1}{\beta} \sum_{\omega}|\omega|\left(\frac{1}{g}|\phi(\omega)|^{2}+g|\theta(\omega)|^{2}\right) \\
& =\frac{g}{2 \beta} \sum_{\omega}|\omega|\left(\left|\theta_{+}(\omega)\right|^{2}+\left|\theta_{-}(\omega)\right|^{2}\right),
\end{aligned}
$$

where $\phi=\Phi(x=0), \theta=\Theta(x=0)$ and $\theta_{ \pm}=\theta \mp \frac{\phi}{g}$. In the back-scattering problem, [32] $\theta$ is free and eventually can be integrated out from the effective action, but let us for the moment keep $\theta$. We need both fields in order to treat 1 . back-scattered current $i_{\text {back }}, 2$. electron injection (ejection) $\vec{i}_{\text {bulk }}$, on the same footing. We will see that (20) is the suitable way of writing $S_{0}$ for the latter problem.

\section{A. Backward scattering}

We first consider the backscattering problem [32]. The scattering potential due to the tunneling of quasiparticle reads

$$
\begin{aligned}
\mathcal{L}_{\text {back }} & \sim \Gamma_{\text {back }}\left[\Psi_{+k_{\mathrm{F}}}^{\dagger} \Psi_{-k_{\mathrm{F}}}+\Psi_{+k_{\mathrm{F}}} \Psi_{-k_{\mathrm{F}}}^{\dagger}\right]_{x=0} \\
& \sim \delta(x) \Gamma_{\text {back }} \cos (2 \sqrt{\pi} \phi),
\end{aligned}
$$

i.e., $\theta$ is free and can be integrated out from the effective action. By throwing away $\theta$-part in (19); the effective action at $x=0$ reduces to

$$
\begin{aligned}
S_{\text {back }}(\Lambda) & =\frac{1}{\beta g} \sum_{|\omega|<\Lambda}|\omega|\left|\phi_{\Lambda}(\omega)\right|^{2} \\
& +\Gamma_{\text {back }}(\Lambda) \int_{0}^{\beta} d \tau \cos \left(2 \sqrt{\pi} \phi_{\Lambda}\right) .
\end{aligned}
$$

This effective theory is meaningless unless we find a suitable high-frequecy cutoff $\Lambda$, i.e., starting from bare cutoff 
$\Lambda_{0}$, we integrate out the high-frequency unphysical degrees of freedom down to $\Lambda$. By decomposing the bosonic field $\phi$ in Fourier space into fast $(\Lambda-d \Lambda<\omega<\Lambda)$ and slow $(0<\omega<\Lambda-d \Lambda)$ parts and then integrating out the high-frequency modes $\Lambda-d \Lambda<\omega<\Lambda$, one obtains the RG equation for $\Gamma_{\text {back }}(\Lambda)$ as $\Gamma_{\text {back }}(\Lambda)=$ $\Gamma_{\text {back }}\left(\Lambda_{0}\right)\left(\frac{\Lambda}{\Lambda_{0}}\right)^{g-1}$. The scaling behavior of backscattered current $i_{\text {back }}$ is deduced from the RG equation by identifying the physical $\Lambda$ to be $\Lambda_{\text {back }}=g e\left(V_{+}-V_{-}\right)=$ $V_{S}-V_{D}$ so that $i_{\text {back }} \sim\left(V_{S}-V_{D}\right)^{2 g-1}$, where we have chosen by convention as $V_{S}>V_{D}$. Notice that in the high-frequency (ohmic) limit $\left(V_{S}-V_{D} \rightarrow \infty\right)$ the correction to the two-terminal conductance vanishes: $\frac{d i_{\text {back }}}{d\left(V_{S}-V_{D}\right)} \sim\left(V_{S}-V_{D}\right)^{2(g-1)} \rightarrow 0$ as long as $0<g<1$.

\section{B. Electron injection (ejection)}

For the tunneling of electron into or out of the bulk sample, we basically follow the same spirit. Through the bulk contact at $x=0$ electrons can tunnel into the bulk sample which has two chirally separated eigen modes respectively at voltages $V_{+}$and $V_{-}$from the electron reservoir at chemical potential $\mathrm{eV}$. An electron incident from this electron reservoir at chemical potential $\mathrm{eV}$ must be decomposed into two fractionally charged quasiparticles in order to be absorbed in the bulk sample. The incident Fermi liquid electron ends up in the final state with one of the two possible electronic excitations of TLL, i.e., either

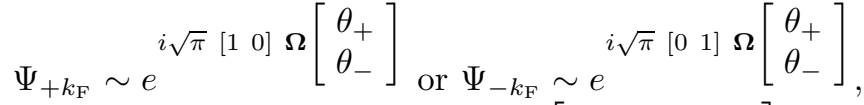
where $\Omega$ has been defined as $\boldsymbol{\Omega}=\frac{1}{2}\left[\begin{array}{ll}1+g & 1-g \\ 1-g & 1+g\end{array}\right]$. The scattering potential due to an injection or ejection of electrons at $x=0$ is given, respectively, for the $+k_{\mathrm{F}^{-}}$and $-k_{\mathrm{F}}$-channel as

$$
\begin{aligned}
\mathcal{L}_{+k_{\mathrm{F}}} & =\Gamma_{+k_{\mathrm{F}}}\left[\Psi_{+k_{\mathrm{F}}}^{\dagger} \Psi_{g=1}+\Psi_{+k_{\mathrm{F}}} \Psi_{g=1}^{\dagger}\right] \\
& \sim \Gamma_{+k_{\mathrm{F}}}\left[\Psi_{g=1}^{\dagger} e^{i \sqrt{\pi}\left(\frac{1+g}{2} \theta_{+}+\frac{1-g}{2} \theta_{-}\right)}+(\text {h.c. })\right] \\
\mathcal{L}_{-k_{\mathrm{F}}} & =\Gamma_{-k_{\mathrm{F}}}\left[\Psi_{-k_{\mathrm{F}}}^{\dagger} \Psi_{g=1}+\Psi_{-k_{\mathrm{F}}} \Psi_{g=1}^{\dagger}\right] \\
& \left.\sim \Gamma_{-k_{\mathrm{F}}}\left[\Psi_{g=1}^{\dagger} e^{i \sqrt{\pi}\left(\frac{1-g}{2} \theta_{+}+\frac{1+g}{2} \theta_{-}\right)}+\text {(h.c. }\right)\right] .
\end{aligned}
$$

The total effective action is

$$
\begin{aligned}
S_{\text {bulk }} & =\frac{g}{2 \beta}\left[\sum_{|\omega|<\Lambda_{+}}|\omega|\left|\theta_{+}(\omega)\right|^{2}+\sum_{|\omega|<\Lambda_{-}}|\omega|\left|\theta_{-}(\omega)\right|^{2}\right] \\
& +\int_{0}^{\beta} d \tau \mathcal{L}_{+k_{\mathrm{F}}}\left[\theta_{+}, \theta_{-}\right]+\int_{0}^{\beta} d \tau \mathcal{L}_{-k_{\mathrm{F}}}\left[\theta_{+}, \theta_{-}\right],
\end{aligned}
$$

where we have introduced two frequency cut-offs $\Lambda_{+}$and $\Lambda_{-}$, respectively, for $\theta_{+}$and for $\theta_{-}$. Notice that in Eq. (25) $S_{0}$ is represented as (20). Now we derive the RG equation for $\Gamma_{+k_{\mathrm{F}}}\left(\Lambda_{+}, \Lambda_{-}\right)$and $\Gamma_{-k_{\mathrm{F}}}\left(\Lambda_{+}, \Lambda_{-}\right)$. using the standard perturbative RG analysis. At leading order the two RG equations are decoupled and can be treated independently. Furthermore the cutoff frequencies $\Lambda_{+}$ and $\Lambda_{-}$can be different for the two RG equations, since available energy shells in $S_{0}$ for (23) and (24) can be differernt. The crucial step is, therefore, to find these cut-off frequencies, i.e., $\Lambda_{+k_{\mathrm{F}}}^{+}, \Lambda_{+k_{\mathrm{F}}}^{-}$for $\Gamma_{+k_{\mathrm{F}}}$, and $\Lambda_{-k_{\mathrm{F}}}^{+}$, $\Lambda_{-k_{\mathrm{F}}}^{-}$for $\Gamma_{-k_{\mathrm{F}}}$. In the Appendix these cutoffs are derived based on a microscopic analysis where the fractionalization of an incident electron is explicit. By simply observing the physical processes in (23), it is however not difficult to convince ourselves that the natural cutoffs for (23) are $\Lambda_{+k_{\mathrm{F}}}^{+}=\Lambda_{+k_{\mathrm{F}}}^{-}=e\left(V-V_{+}^{(0)}\right)$, where $V_{+}^{(0)}=$ $\frac{1+g}{2} e V_{+}+\frac{1-g}{2} e V_{-}$is the chemical potential of a $+k_{\mathrm{F}}$ electron incident from two chiral eigenmodes of TLL. Thus the RG equation for $\Gamma_{+k_{\mathrm{F}}}$ reduces to that of a single scaling parameter $\Lambda$, i.e., following the same procedure as the backscattering problem, one can find the RG equation for $\Gamma_{+k_{\mathrm{F}}}(\Lambda)$ as $\Gamma_{+k_{\mathrm{F}}}(\Lambda)=\Gamma_{+k_{\mathrm{F}}}\left(\Lambda_{0}\right)\left(\frac{\Lambda}{\Lambda_{0}}\right)^{\Delta+1 / 2-1}$, where $\Delta=\frac{1}{4}\left(g+\frac{1}{g}\right)$ is nothing but the anomalous scaling dimension of a TLL electron operator. The scaling behavior of bulk injected current $i_{+k_{F}}$ is deduced from the RG equation by identifying the physical $\Lambda$ to be $\Lambda=\Lambda_{+k_{\mathrm{F}}}^{+}=\Lambda_{+k_{\mathrm{F}}}^{-}=e\left(V-V_{+}^{(0)}\right)$ as

$$
i_{+k_{\mathrm{F}}} \sim\left\{\begin{array}{l}
\left(V-V_{+}^{(0)}\right)^{2 \Delta} \quad \text { for } V>V_{+}^{(0)} \\
-\left(V_{+}^{(0)}-V\right)^{2 \Delta} \quad \text { for } V<V_{+}^{(0)}
\end{array} .\right.
$$

Note that the energy dependence of Eq. (26) is not a simple product of two chiral components such as $\left(V-V_{+}\right)^{\Delta}\left(V-V_{-}\right)^{\Delta}$ as might be naively expected. The same argument applies for the RG equation for $\Gamma_{-k_{\mathrm{F}}}$. The scaling behavior of bulk injected current $i_{-k_{\mathrm{F}}}$ is obtained as

$$
i_{-k_{\mathrm{F}}} \sim\left\{\begin{array}{l}
\left(V-V_{-}^{(0)}\right)^{2 \Delta} \quad \text { for } V>V_{-}^{(0)} \\
-\left(V_{-}^{(0)}-V\right)^{2 \Delta} \quad \text { for } V<V_{-}^{(0)}
\end{array},\right.
$$

where $e V_{-}^{(0)}=\frac{1-g}{2} e V_{+}+\frac{1+g}{2} e V_{-}$is the chemical potential of $-k_{\mathrm{F}}$ electron. In the absence of stationary current $I_{\text {bias }}$, both Eqs. (26) and (27) reduce to the trivial scaling law: $i_{+k_{\mathrm{F}}}=i_{-k_{\mathrm{F}}} \sim \pm\left|V-V_{0}\right|^{2 \Delta}$, depending on the sign of $V-V_{0}$.

\section{Conductances}

We now turn to the discussion of the physical consequences of Eqs. (14, 15) and Eqs. (16, 17), which correspond, respectively, to the boundary conditions discussed in Secs. III-A and III-B. In Secs V-A and V-B, in particular, in Eqs. (26) and (27), we were able to relate the 
bulk injected currents $i_{ \pm k_{\mathrm{F}}}$ to local energy scales in the vicinity of the bulk contact such as $V-V_{+}^{(0)}$ or $V-V_{-}^{(0)}$. In order to compare this term with other contributions one has to rewrite them in terms of the source-drain voltage $V_{S}-V_{D}$. We focus on the case where the two end contacts impose a large stationary current $I_{\text {bias }}$ in the quantum wire so that

1. The chiral symmetry of the system should be preserved.

2. The additional current injected through the bulk contact can be treated as a small perturbation.

We will see that under the two boundary conditions discussed in Sec. III-A and Sec. III-B, the 3T SD conductance $G_{\mathrm{SD}}^{(3)}$ interpolates between the two fixed points: $G_{\mathrm{SD}}=e^{2} / h$ and $G_{\mathrm{SD}}=g e^{2} / h$ in the ohmic limit. We found non-trivial scaling exponents as a result of specific boundary condition and electron fractionalization.

\section{One bulk contact with end contacts in equilibrium with bare electrons}

In the case of equilibration with bare electrons at the end contacts (see section III-A), i.e., in the case of $\left(14,15\right.$, due to the boundary condition (9), $V_{+}^{(0)}$ in $(26)$ and $V_{-}^{(0)}$ in (27) can be replaced, at leading order, by $V_{+}^{(0)}=V_{S}$ and $V_{-}^{(0)}=V_{D}$, respectively. Now let us consider the case of Eq. (14), in which the bulk contact is connected to the source $(\mathrm{S})$ through an ohmic resistance $r_{S}$. We are mainly interested in the case: $V_{D} \ll V=V_{S}-r_{S} i_{\text {bulk }}<V_{S}$, i.e., in the case of small $i_{\text {bulk }}>0$, since this gives the first correction to the "endcontact model". Substituting Eq. (26) into Eq. (14), one can immediately see that the contribution to the total current $I$ of the bulk injected current obeys a power law with an exponent $2 \Delta$. The important result of our RG analysis, which is carefully derived in Sec. V-A and $\mathrm{V}-\mathrm{B}$ as well as in the Appendix, is that this exponent appears as a power of the voltage difference $V_{S}-V$ and not of the source-drain voltage $V_{S}-V_{D}$.

Now we have to rewrite this energy scale $V_{S}-V$ in terms of $V_{S}-V_{D}$. Let us notice that $i_{+k_{\mathrm{F}}} \sim-\left(V_{S}-V\right)^{2 \Delta}$ is negative and very small when $V_{D} \ll V<V_{S}$ since $2 \Delta=\frac{1}{2}\left(g+\frac{1}{g}\right) \geq 1$. On the other hand, $i_{-k_{\mathrm{F}}} \sim$ $\left(V-V_{D}\right)^{2 \Delta} \sim\left(V_{S}-V_{D}\right)^{2 \Delta}$ gives a positive dominant contribution to $i_{\text {bulk }}$ compensating for the negative $i_{+k_{\mathrm{F}}}$ to ensure a positive $i_{\mathrm{bulk}}=i_{+k_{\mathrm{F}}}+i_{-k_{\mathrm{F}}}$. Thus the small voltage difference $V_{S}-V$ which appears in the scaling of $i_{+k_{\mathrm{F}}}$ can be rewitten as a function of the bias voltage $V_{S}-V_{D}$ as $V_{S}-V \sim i_{\text {bulk }} \sim i_{-k_{\mathrm{F}}} \sim\left(V_{S}-V_{D}\right)^{2 \Delta}$. In other words, $V_{S}-V$ itself scales with the exponent $2 \Delta$ in terms of the source-drain voltage $V_{S}-V_{D}$. The combination of these two $2 \Delta$ 's leads to an unusual scaling behavior of the conductance measured in terms of the bias voltage $V_{S}-V_{D}$ :

$$
G_{\mathrm{SD}}^{(3)}=\frac{e^{2}}{h}-c_{1}\left(V_{S}-V_{D}\right)^{2(g-1)}-c_{2}\left(V_{S}-V_{D}\right)^{(2 \Delta)^{2}-1},
$$

where $c_{1}$ and $c_{2}$ are scale-invariant positive constants. Eq. (28) constitutes one of the main results of the paper, since it not only contains a new type of scaling behavior $\left(V_{S}-V_{D}\right)^{(2 \Delta)^{2}-1}$ but also justifies our model as a possible way to interpolate between the two boundary conditions. Notice that $(2 \Delta)^{2}-1 \geq 0$ for $0<g \leq 1$. Eq. (28) means that even in the ohmic regime where the backscattering current $i_{\text {back }}$ scales to zero, the twoterminal conductance in the presence of the bulk contact can be decreased due to the bulk-injected current $i_{\text {bulk }}$ and interaction $(0<g<1)$.

In the case of the bulk contact connected to the drain (D), i.e., Eq. (15), one finds, of course, the same universal behavior as (28) as far as one focuses on the case $V_{D}<V \ll V_{S}$, i.e., the leading order correction to the boundary condition (3).

\section{One bulk contact with end contacts in equilibrium with dressed eigenmodes}

Let us now consider the second boundary condition for the end contacts: $V_{S}=V_{R}^{+}, V_{D}=V_{L}^{-}$, i.e., we discuss the scaling behavior of $(16,17)$. In this case, depending on whether the bulk contact is connected to the source (S) or to the drain (D) all the $V$ 's in Eqs. (A1), A2 can be replaced either by $V=V_{+}$or by $V=V_{-}$. Then one can, of course, replace $V_{+}$and $V_{-}$respectively, by $V_{S}$ and by $V_{D}$. Substituting $V=V_{+}=V_{S}, V_{-}=V_{D}$ into Eqs. (A1, A2 , one finds $\Lambda_{+k_{\mathrm{F}}}^{+}=\Lambda_{+k_{\mathrm{F}}}^{-}=\frac{1-g}{2}\left(V_{S}-V_{D}\right)$ as well as $\Lambda_{-k_{\mathrm{F}}}^{+}=\Lambda_{-k_{\mathrm{F}}}^{-}=\frac{1+g}{2}\left(V_{S}-V_{D}\right)$. Eqs. (26), (27) may also be rewritten accordingly. Taking into account that $i_{+}=\frac{1+g}{2} i_{+k_{\mathrm{F}}}+\frac{1-g}{2} i_{-k_{\mathrm{F}}}>0$ in contrast to the previous case: $i_{+k_{\mathrm{F}}}<0$, the leading scaling behavior of Eq. (16) is found to be

$$
G_{\mathrm{SD}}^{(3)}=g \frac{e^{2}}{h}-c_{1}\left(V_{S}-V_{D}\right)^{2(g-1)}+c_{3}\left(V_{S}-V_{D}\right)^{2 \Delta-1},
$$

where $c_{1}$ and $c_{3}$ are scale-invariant positive constants. Comparing with (28), one can observe that in Eq. (29),

1. The existence of the bulk current increases $G_{\mathrm{SD}}^{(3)}$, indicating that the correction indeed interpolates between the two limiting cases, i.e., from $G_{\mathrm{SD}}=$ $g e^{2} / h$ to $G_{\mathrm{SD}}=e^{2} / h$.

2. The correction due to the bulk current does not scale in the same way. Note that the exponent of Eq. (29) is simply related to the anomalous scaling dimension of a TLL electron operator. 


\section{Remarks on energy scales}

Before ending this section, we would like to make some remarks on the energy scales where Eqs. (28,29) are valid.

1. We have neglected the backscattering at the interface between the 1D sample and the reservoirs. This is justified when $e\left(V_{S}-V_{D}\right) \gg \Lambda_{\text {end }}$, where $\Lambda_{\text {end }}$ is a crossover energy associated with the quasiparticle $\leftrightarrow$ electron tunneling duality model at effective filling factor $\nu_{\text {eff }}$ [25,33.

2. In order for Eqs. 22, 29) to be valid it is also required that backscatterings in the bulk (at the bulk contact or due to some impurities in the bulk) can be treated perturbatively. This situation is achieved when $e\left(V_{S}-V_{D}\right) \gg \Lambda_{\text {back }}$, where $\Lambda_{\text {back }}$ is another crossover energy scale: $\Lambda_{\text {back }} \propto$ $\Gamma_{\text {back }}\left(\Lambda_{0}\right)^{1 /(1-g)}$. 32

3. Finally $V_{S}-V_{D}$ must be sufficiently large so that the perturbative analysis for the electron injection (ejection) can be justified, i.e., $e\left(V_{S}-V_{D}\right) \gg \Lambda_{\text {bulk }}$, where $\Lambda_{\text {bulk }} \propto \Gamma_{ \pm k_{F}}\left(\Lambda_{0}\right)^{2 /(1-2 \Delta)}, \Delta=\frac{1}{4}\left(g+\frac{1}{g}\right)$.

We have also assumed that

1. the temperature $T$ is sufficiently low,

2. the typical size $L$ of the system is large enough,

so that $e\left(V_{S}-V_{D}\right) \gg T, u / L$ should be satisfied, where $u=v_{F} / g$ is the velocity of the chiral eigen modes.

We studied the effects of one bulk contact as a leading order correction to the two limiting cases of end contact model: (A) $G_{\mathrm{SD}}=e^{2} / h$ and $(\mathrm{B}) G_{\mathrm{SD}}=g e^{2} / h$, corresponding, respectively, to the $(\mathrm{A})$ the presence and $(\mathrm{B})$ the absence of screening by a metallic gate discussed in Sec. III. We found,

1. In both cases $G_{\mathrm{SD}}^{(3)}$ is not quantized even in the ohmic limit $V_{S}-V_{D} \rightarrow \infty$, interpolating between the two limiting cases: (A) $G_{\mathrm{SD}}=e^{2} / h$ and (B) $G_{\mathrm{SD}}=g e^{2} / h$.

2. The correction due to the bulk current, however, does not scale in the same way (see Eqs. (28) and (29)). In the presence of screening metallic gate, i.e., in the case of Eq. (28), it exhibits a pronounced scaling behavior: $\left(V_{S}-V_{D}\right)^{(2 \Delta)^{2}-1}$, where $2 \Delta=\frac{1}{2}\left(g+\frac{1}{g}\right)>1(2 \Delta=1)$ for (non) interacting case.

In terms of the RG picture introduced at the beginning of this section, UV-relevant deviation from the standard two fixed points: (A) $G_{\mathrm{SD}}=e^{2} / h$ and (B) $G_{\mathrm{SD}}=g e^{2} / h$ may suggest a possibility of new continuous fixed points between the above two values.

\section{GENERALIZATION TO MANY BULK CONTACTS}

In this section we continue the analysis of the bulk contacts. Some of the electrons are injected into the nanotube through $N_{L}$ bulk contacts in the left reservoir (others are through the end contact). Both of them contribute to the total current $I$ which flows through the sample. Similarly in the right reservoir some electrons are ejected from the nanotube not only at both ends but also through one of the $N_{R}$ bulk contacts. In brief we generalize the analysis of one bulk contact to many bulk contacts.

This model is clearly inspired by the work of Chamon and Fradkin. The curious result derived in Ref. 25] is that the conductance is not monotonous as a function of $N_{R}$ and $N_{L}$ (See Sec. III-C), and has a sort of damped oscillatory behaviour which depends on the parities of $N_{R}$ and $N_{L}$. 40] So we were curious to determine if such a non-monotonous behaviour would also be predicted in the case of the non-chiral TLL, and if the renormalized conductance could be experimentally measured in the case of many contacts, as opposed to the situation found in [5]7. As will appear below, our answer is that the non-chiral TLL does not behave like a chiral one as far as the number of contacts is concerned, although the conductance results are affected when weak contacts are applied in the bulk of the sample strongly connected to reservoir through its end points.

Another motivation behind this type of model is the experiment by Kasumov et. al. [17], where they found a clear signature of superconduting behavior in isolated SWNT samples as well as nanotube ropes. In this experiment the isolated SWNT sample $S T_{1}$ exhibits a resistance $R$ which saturates at high temperatures to $R \sim 25 \mathrm{k} \Omega$ (see Fig. 2B of Ref. [17]). As has been already mentioned, this corresponds to the conductance $G_{\mathrm{SD}} \sim e^{2} / h$, which is smaller by a factor $1 / 4$ than $G_{\mathrm{SD}}=4 e^{2} / h$ expected from the boundary condition (3). In this experiment the nanotubes are embedded (melt) into the reservoirs at both ends. Given the finite radius of the nanotube $(\sim 1.5 \mathrm{~nm})$ and possible roughness of the electrode surface from which the nanotube emerges, it is legitimate to question the validity of single point contact model. What is hoped here is that our model with a weak bulk contact is a first step towards a proper description of this experimental situation.

\section{A. Many bulk contacts with end contacts in equilibrium with bare electrons}

Consider $N_{R}+N_{L}$ independent bulk contacts $N_{L}$ of which are connected to the source $(\mathrm{S})$ and the rest of which to the drain (D). The boundary condition (9) is generalized to 


$$
\left\{\begin{array}{l}
V_{S}=V_{-N_{L}}^{+(0)}=\left[\begin{array}{ll}
1 & 0
\end{array}\right] \boldsymbol{\Omega} \vec{V}_{-N_{R}} \\
V_{D}=V_{N_{R}}^{-(0)}=\left[\begin{array}{ll}
0 & 1
\end{array}\right] \boldsymbol{\Omega} \vec{V}_{N_{L}}
\end{array}\right.
$$

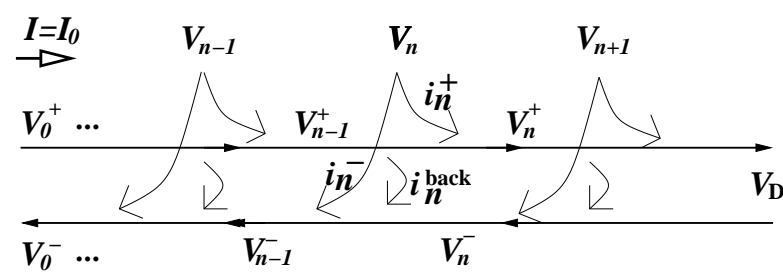

FIG. 2. Many bulk contacts.

Let us focus on the $n$th bulk contact $\left(n=1, \cdots, N_{R}\right)$, which is, by definition, connected to the drain (D). The voltage equations at $n$th bulk contact can be written as $i_{n}^{+}-i_{n}^{\text {back }}=g \frac{e^{2}}{h}\left(V_{n-1}^{+}-V_{n}^{+}\right), i_{n}^{-}+i_{n}^{\text {back }}=$ $g \frac{e^{2}}{h}\left(V_{n}^{-}-V_{n-1}^{-}\right) . \quad i_{n}^{+}\left(i_{n}^{-}\right)$is a current injected into the TLL eingenmode with $+(-)$-chirality. The definition of eigenmode voltages $V_{n}^{ \pm}$are given in Fig. 2. Using the vector notation $\vec{i}_{n}=\left[\begin{array}{c}i_{n}^{+} \\ i_{n}^{-}\end{array}\right], \vec{V}_{n}=\left[\begin{array}{c}V_{n}^{+} \\ V_{n}^{-}\end{array}\right]$, one may rewrite the voltage drop equation as $\vec{V}_{n-1}-\vec{V}_{n}=$ $\frac{\sigma_{\mathbf{z}} \vec{i}_{n} \text { bulk }}{g e^{2} / h}-\frac{i_{n}^{\text {back }}}{g e^{2} / h}\left[\begin{array}{l}1 \\ 1\end{array}\right]$. The total current circulating in the system is defined as $I=I_{0}=[1,-1] \Omega \vec{V}_{0}$. Using recursively the voltage equation, one finds $V_{N_{R}}=$ $\vec{V}_{0}+\sum_{n=1}^{N_{R}}\left(\frac{\sigma_{\mathbf{z}} \vec{i}_{n}^{\text {bulk }}}{g e^{2} / h}-\frac{i_{n}^{\text {back }}}{g e^{2} / h}\left[\begin{array}{l}1 \\ 1\end{array}\right]\right)$. Following the same procedure, one will find a similar relation for the left contact: $V_{-N_{L}}=\vec{V}_{0}-\sum_{n=1}^{N_{L}}\left(\frac{\sigma_{\mathbf{z}} \vec{i}_{-n}^{\text {bulk }}}{g e^{2} / h}-\frac{i_{-n}^{\text {back }}}{g e^{2} / h}\left[\begin{array}{l}1 \\ 1\end{array}\right]\right)$.

Using recursively these voltage drop equations, one finds

$$
\begin{aligned}
& V_{S}=\left[\begin{array}{ll}
1 & 0
\end{array}\right] \boldsymbol{\Omega}\left[\vec{V}_{0}-\sum_{n=1}^{N_{L}}\left(\frac{\sigma_{\mathbf{z}} \vec{i}_{-n}^{\text {bulk }}}{g e^{2} / h}-\frac{i_{-n}^{\text {back }}}{g e^{2} / h}\left[\begin{array}{l}
1 \\
1
\end{array}\right]\right)\right], \\
& V_{D}=\left[\begin{array}{ll}
0 & 1
\end{array}\right] \boldsymbol{\Omega}\left[\vec{V}_{0}+\sum_{n=1}^{N_{R}}\left(\frac{\sigma_{\mathbf{z}} \vec{i}_{n}^{\text {bulk }}}{g e^{2} / h}-\frac{i_{n}^{\text {back }}}{g e^{2} / h}\left[\begin{array}{l}
1 \\
1
\end{array}\right]\right)\right] .
\end{aligned}
$$

Thus the bias voltage $V_{S}-V_{D}$ can be expressed in terms of $\vec{V}_{0}, i_{ \pm n}^{\text {back }}$ and $\vec{i}_{ \pm n}$ as

$$
\begin{gathered}
V_{S}-V_{D}=[1,-1] \boldsymbol{\Omega} \vec{V}_{0}+\sum_{n=1}^{N_{L}} \frac{i_{-n}^{\text {back }}}{g e^{2} / h}+\sum_{n=1}^{N_{R}} \frac{i_{n}^{\text {back }}}{g e^{2} / h} \\
-\left[\begin{array}{ll}
1 & 0
\end{array}\right] \boldsymbol{\Omega} \sigma_{\mathbf{z}} \sum_{n=1}^{N_{L}} \frac{\vec{i}_{-n}^{\text {bulk }}}{g e^{2} / h}-\left[\begin{array}{ll}
0 & 1
\end{array}\right] \boldsymbol{\Omega} \sigma_{\mathbf{z}} \sum_{n=1}^{N_{R}} \frac{\vec{i}_{n}^{\text {bulk }}}{g e^{2} / h}
\end{gathered}
$$

Recall that $\boldsymbol{\Omega} \sigma_{\mathbf{z}} \boldsymbol{\Omega}=g \sigma_{\mathbf{z}}$. Using the decomposition analogous to (7), one can see that $i_{-n}^{-k_{F}}\left(n=1, \cdots, N_{L}\right)$ and $i_{n}^{+k_{\mathrm{F}}}\left(n=1, \cdots, N_{R}\right)$ do not contribute to (31). The total current circulating in the system is $I=I_{0}=[1,-1] \Omega \vec{V}_{0}$. Since we are interested in the first correction to (3), we consider the case where all the bulk contacts connected to the source (S) are at voltages $V_{D}<V \ll V_{S}$ and all the bulk contacts connected to the drain (D) are at voltages $V_{D} \ll V<V_{S}$. If this is the case, the leading scaling behavior of the conductance in terms of the bias voltage $V_{S}-V_{D}$ obtained from Eq. (31) reduces to (28) independently of $N_{R}$ and $N_{L}$. Eq. (28), therefore, the non-chiral version of [40] does not exhibit an oscillatory behavior as a function of $\left(N_{R}, N_{L}\right)$. Nevertheless Eq. (28) indeed interpolates between the two boundary conditions discussed in Sec. III, which was also the case in [40].

\section{B. Many bulk contacts with end contacts in equilibrium with dressed eigenmodes}

In the case of equilibration with dressed eigenmodes the boundary condition (30) should be replaced by $V_{S}=$ $V_{-N_{R}}^{+}, V_{D}=V_{N_{L}}^{-}$. Using the same voltage drop equations, one can easily see, using the decomposition analogous to (7), that $i_{-n}^{-}\left(n=1, \cdots, N_{R}\right)$ and $i_{n}^{+}(n=$ $\left.1, \cdots, N_{L}\right)$ do not contribute to the total current $I=$ $I_{0}=g \frac{e^{2}}{h}[1,-1] \vec{V}_{0}$. Then the leading scaling behavior of the conductance in terms of the bias voltage reduces to (29) independently of $N_{R}$ and $N_{L}$.

\section{TLL WITH INTERNAL DEGREES OF FREEDOM - APPLICATION TO SWNT AND MWNT}

Up to now we have considered for simplicity spinless TLL model, where $2 \Delta=\frac{1}{2}\left(g+\frac{1}{g}\right)$. In order to apply the above results, in particular, Eqs. (28) and (29) for nanotubes let us recall the following properties of SWNT and MWNT: The SWNT have four conducting channels: two subbands $\times$ (charge, spin) at roomtemperatures, indicating an expected quantized conductance: $G_{\mathrm{SD}}=4 e^{2} / h$.

The experimental data for conductance measurements in SWNT and MWNT display a variety of results. The ballistic transport in carbon nanotubes was first observed in MWNT, showing the conductance $G_{\mathrm{SD}} \sim 2 e^{2} / h[22$. This implies that in MWNT only one of the two subband contributes to the electronic transport. In the case of SWNT, the observed conductance exhibits fluctuations versus Fermi energy approaching to the theoretically expected value: $G_{\mathrm{SD}}=4 e^{2} / h$ as the temperature is lowered. 16]

Let us now focus on the case of SWNT. The spinless TLL theory studied in earlier sections should be generalized to acquire $2 \times 2=4$ flavors $f=c+, c-, s+, s-$. The four channels are obtained from combining charge $(c)$ and spin $(s)$ degrees of freedom as well as symmetric $(+)$ and anti-symmetric $(-)$ linear combinations of the two Fermi points. Correspondingly we must introduce 
four TLL parameters: $g_{c+}, g_{c-}, g_{s+}, g_{s-}$. The scaling dimension $\Delta$ of TLL electron operator can be written, for example, in terms of these TLL parameters as $\Delta_{\mathrm{SWNT}}=$ $\frac{1}{16} \sum_{f}\left(g_{f}+\frac{1}{g_{f}}\right)$. Whereas the charge conductance $G_{\mathrm{SD}}$ is determined only by $g_{c+}$, i.e., $G_{\mathrm{SD}}=4 g_{c+} e^{2} / h$, as was the case for TLL with spin [41]. In any case one can verify by carefully investigating the effective Coulomb interaction in SWNT 21 that the interaction gives rise to a significant renormalization only for $g_{c+}$, whereas $g_{f} \sim 1$ for $f=c-, s+, s-$ (neutral modes). Thus the TLL parameter $g$ for SWNT is defined as $g=g_{c+}$, which is estimated to be typically in the range $0.2-0.3$.

To summarize one has to make the following replacements in order to apply Eqs. 28) and (29) for SWNT:

1. The anomalous scaling dimension $\Delta$ of TLL electron operator should be replaced by $\Delta_{\mathrm{SWNT}}=$ $\frac{1}{16}\left(g+\frac{1}{g}\right)+\frac{3}{8}$.

2. The ohmic conductance in equilibrium either with bare electrons $\left(G_{\mathrm{SD}}^{(2)}=e^{2} / h\right)$ or with dressed eigen modes $\left(G_{\mathrm{SD}}^{(2)}=g e^{2} / h\right)$ should be multiplied by 4 , in order to accont for the number of conducting channels.

Apart from these changes, however, the main claims of the preceding sections remain unchanged.

\section{DISCUSSION AND CONCLUSIONS}

In the first half of this paper we argued that in the case of standard end-contact geometry, the two-terminal conductance $G_{\mathrm{SD}}^{(2)}$ in the ohmic limit can be either $G_{\mathrm{SD}}^{(2)}=$ $e^{2} / h$ or $G_{\mathrm{SD}}^{(2)}=g e^{2} / h$ depending on the boundary conditions. In our point of view, different boundary conditions apply in the presence or absence of a metallic gate close to the $1 \mathrm{D}$ sample.

It is plausible that the boundary conditions studied in refs. 15 7,29 31 are realized when a gate is present close enough to the wire. This is suggested by the agreement between the result of Ref. [9] and the theoretical analysis using boundary conditions such that bare reservoir electrons are not in equilibrium with dressed eigenmode of the TLL, but with bare particles inside the TLL.

On the other hand, more recent experiments suggest that a variety of other boundary conditions are realized experimentally $12,14,17$. It is tempting to interpret the result of Kasumov et. al. [17] as a consequence of boundary conditions such that bare reservoir electrons are in equilibrium with dressed TLL eigenmodes.

The absence of a gate in this experiment suggests that long-range interactions inside the carbon nanotube are instrumental in bringing about this different boundary condition. If our analysis is correct, a check would be to measure the nanotube conductance in the presence of a metallic gate sufficiently close to the nanotube compared with the sample length for the interactions to be screened. Then we would expect $G_{\mathrm{SD}}^{(2)}=4 e^{2} / h$. It is striking that the result of Kasumov et. al., if interpreted as $G_{\mathrm{SD}}=4 g e^{2} / h$, where $g$ would be the TLL interaction parameter, yields a value $g \sim 0.25$ in very good agreement with the theoretical value calculated in Ref. [21]. Other experiments 12 14] clearly suggest other boundary conditions 26].

Now the nature of two fixed points was understood as different boundary conditions at the end contacts by making clear distinction between the bare and dressed eigenmode densities in the bosonized formulation. In the second half of the paper we proposed a system of 1D sample coupled to bulk contacts as well as end contacts where we found qualitatively different behaviors of the conductance, e.g., different scaling dimensions, as a consequence of a large stationary current. As a result, we found that the addition of bulk contacts interpolates between the two fixed points. The RG analysis for this problem has been developped by taking into account explicitly the fractionalization of electronic charge.

We studied in particular the leading scaling behavior of the corrections to the two-terminal conductance $G_{\mathrm{SD}}^{(3)}$ in the presence of bulk-injected current in (A) the presence and (B) the absence of screening by a metallic gate. We found in both cases $G_{\mathrm{SD}}^{(3)}$ is not quantized even in the ohmic limit $V_{S}-V_{D} \rightarrow \infty$, interpolating between the two limiting cases: (A) $G_{\mathrm{SD}}=e^{2} / h$ and (B) $G_{\mathrm{SD}}=g e^{2} / h$. The correction due to the bulk current, however, does not scale in the same way (see Eqs. (28) and (29)). In the case of Eq. (28), corresponding to the equilibration with bare electrons (Sec. III-A), it exhibits, as a consequence of this particular boundary condition (3)) or more precisely Eq. (9)), a pronounced scaling behavior: $\left(V_{S}-V_{D}\right)^{(2 \Delta)^{2}-1}$, where $2 \Delta=\frac{1}{2}\left(g+\frac{1}{g}\right)$. The understanding of strong coupling limit for the bulk contact was left for future study. The discussion on the shotnoise spectrum under a variety of boundary conditions discussed in this paper is obviously of interest. This will be discussed in a forthcoming publication [26].

\section{ACKNOWLEDGMENTS}

We are grateful to Hélène Bouchiat and Inès Safi for useful discussions. K. I. is supported by JSPS Postdoctoral Fellowships for Research Abroad.

\section{APPENDIX A: RG ANALYSIS FOR FRACTIONAL PARTICLES}

We derive the RG equation for $\Gamma_{+k_{\mathrm{F}}}\left(\Lambda_{+}, \Lambda_{-}\right)$and $\Gamma_{-k_{\mathrm{F}}}\left(\Lambda_{+}, \Lambda_{-}\right)$starting from the effective action 25 with 
$(23,24)$. At leading order the two RG equations are decoupled and can be treated independently. The cutoff frequencies $\Lambda_{+}$and $\Lambda_{-}$can be different for the two RG equations, since available energy shells in $S_{0}$ for (23) and (24) can be different. The crucial step was, therefore, to find these cut-off frequencies, i.e., $\Lambda_{+k_{\mathrm{F}}}^{+}, \Lambda_{+k_{\mathrm{F}}}^{-}$for $\Gamma_{+k_{\mathrm{F}}}$, and $\Lambda_{-k_{\mathrm{F}}}^{+}, \Lambda_{-k_{\mathrm{F}}}^{-}$for $\Gamma_{-k_{\mathrm{F}}}$.

When an electron incident from the electron reservoir at chemical potential $e V$ tunnels into the TLL, it must be decomposed into two fractionally charged quasiparticles in order to be absorbed in the bulk sample. This final state TLL electron has either of the following energies $e V_{+}^{(0)}=\left[\begin{array}{ll}1 & 0\end{array}\right] \boldsymbol{\Omega}\left[\begin{array}{l}e V_{+} \\ e V_{-}\end{array}\right], e V_{-}^{(0)}=\left[\begin{array}{ll}0 & 1\end{array}\right] \boldsymbol{\Omega}\left[\begin{array}{l}e V_{+} \\ e V_{-}\end{array}\right]$, respectively, for $\Gamma_{+k_{\mathrm{F}}}$ and for $\Gamma_{-k_{\mathrm{F}}}$. In contrast the energy decomposition of the incident Fermi liquid electron is quite arbitrary, i.e., $e V=\left[\begin{array}{ll}1 & 0\end{array}\right] \boldsymbol{\Omega}\left[\begin{array}{l}e V_{+}^{\prime} \\ e V_{-}^{\prime}\end{array}\right]$ for $\Gamma_{+k_{\mathrm{F}}}$ and $e V=\left[\begin{array}{ll}0 & 1\end{array}\right] \boldsymbol{\Omega}\left[\begin{array}{l}e V_{+}^{\prime} \\ e V_{-}^{\prime}\end{array}\right]$ for $\Gamma_{-k_{\mathrm{F}}}$, where $V_{+}^{\prime}\left(V_{-}^{\prime}\right)$ is a part of the electrostatic potential attributed to the $+(-)$ chirality. The only constraint is that both $V_{+}^{\prime}-V_{+}$and $V_{-}^{\prime}-V_{-}$should have the same sign, i.e., they are positive (negative) when the current is injected (ejected). Taking into account this constraint, one can count the available energy shells for tunneling. These procedures are schematically explained in Fig. 3 .

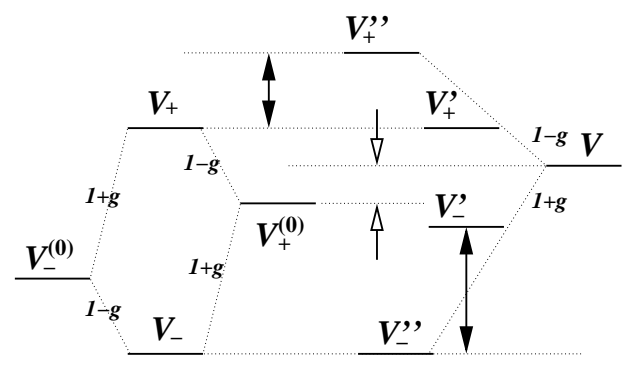

FIG. 3. Available energy shells for the tunneling into TLL.

Let us focus on the RG equation for $\Gamma_{+k_{F}}$, i.e., for the tunneling of $+k_{\mathrm{F}}$ electron. On the left it is shown that the final state TLL electron has either of the following energies $e V_{+}^{(0)}=\left[\begin{array}{ll}1 & 0\end{array}\right] \boldsymbol{\Omega}\left[\begin{array}{l}V_{+} \\ V_{-}\end{array}\right], e V_{-}^{(0)}=\left[\begin{array}{ll}0 & 1\end{array}\right] \boldsymbol{\Omega}\left[\begin{array}{l}V_{+} \\ V_{-}\end{array}\right]$, respectively, for $\Gamma_{+k_{\mathrm{F}}}$ and for $\Gamma_{-k_{\mathrm{F}}}$. On the right we focus on the tunneling of $+k_{\mathrm{F}}$ electron incident from the bulk contact at chemical potential $\mathrm{eV}$. We consider the case $V_{+}^{(0)}<V<V_{+}$. The energy of the incident elecron is decomposed into each chirality as $e V=\left[\begin{array}{ll}1 & 0\end{array}\right] \boldsymbol{\Omega}\left[\begin{array}{l}e V_{+}^{\prime} \\ e V_{-}^{\prime}\end{array}\right]$. In order for a current to be injected, $V_{+}^{\prime} \geq V_{+}$must be satisfied. When $V_{+}^{\prime}=V_{+}, V-V_{-}^{\prime}=\frac{1+g}{1-g}\left(V_{+}-V\right)$. Starting from this value, $V_{-}^{\prime}$ can take values down to $V_{-}$. When $V_{-}^{\prime}$ reaches this limit, where we redefine the energy decomposition of the incident electron as $e V=\left[\begin{array}{ll}1 & 0\end{array}\right] \boldsymbol{\Omega}\left[\begin{array}{l}e V_{+}^{\prime \prime} \\ e V_{-}^{\prime \prime}\end{array}\right]$, therefore, $V_{-}^{\prime \prime}=V_{-}$, the other $V_{+}^{\prime \prime}$ satisfies $V_{+}^{\prime \prime}-V=\frac{1-g}{1+g}\left(V-V_{-}\right)$. The cutoff energy scales which appear in the RG equation for $\Gamma_{+k_{\mathrm{F}}}$ are determined as $\Lambda_{+k_{\mathrm{F}}}^{+}=\frac{1+g}{2} e\left(V_{+}^{\prime \prime}-V_{+}\right), \Lambda_{+k_{\mathrm{F}}}^{-}=\frac{1-g}{2} e\left(V_{-}^{\prime}-V_{-}\right)$.

Thus we were able to derive on microscopic grounds the energy cutoffs which we have used to find (26),

$$
\begin{aligned}
\Lambda_{+k_{\mathrm{F}}}^{+} & =\frac{1+g}{2} e\left[\frac{1-g}{1+g}\left(V-V_{-}\right)-\left(V_{+}-V\right)\right] \\
& =e\left(V-V_{+}^{(0)}\right), \\
\Lambda_{+k_{\mathrm{F}}}^{-} & =\frac{1-g}{2} e\left[\left(V-V_{-}\right)-\frac{1+g}{1-g}\left(V_{+}-V\right)\right] \\
& =e\left(V-V_{+}^{(0)}\right),
\end{aligned}
$$

i.e., $\Lambda_{+k_{\mathrm{F}}}^{+}=\Lambda_{+k_{\mathrm{F}}}^{-}$. Once these energy scales are determined, one can employ the RG analysis for $\Gamma_{+k_{\mathrm{F}}}$.

The same argument applies for the RG equation for $\Gamma_{-k_{\mathrm{F}}}$. The cutoff frequencies for the tunneling of $-k_{\mathrm{F}}$ electron are obtained as

$$
\begin{aligned}
\Lambda_{-k_{\mathrm{F}}}^{+} & =\frac{1-g}{2} e\left[\frac{1+g}{1-g}\left(V-V_{-}\right)-\left(V_{+}-V\right)\right] \\
& =e\left(V-V_{-}^{(0)}\right) \\
\Lambda_{-k_{\mathrm{F}}}^{-} & =\frac{1+g}{2} e\left[\left(V-V_{-}\right)-\frac{1-g}{1+g}\left(V_{+}-V\right)\right] \\
& =e\left(V-V_{-}^{(0)}\right)
\end{aligned}
$$

Hence the RG equation for $\Gamma_{-k_{F}}$ takes the same form as that of $\Gamma_{+k_{\mathrm{F}}}$ except that one should identify $\Lambda$ to be $\Lambda=\Lambda_{-k_{\mathrm{F}}}^{+}=\Lambda_{-k_{\mathrm{F}}}^{-}=V-V_{-}^{(0)}$.

[1] K.-V. Pham, M. Gabay and P. Lederer, Phys. Rev. B 61, 16397 (2000).

[2] V. J. Goldman and B. Su, Science 267, 1010-1012 (1995); V. J. Goldman, I. Karakurt, Jun Liu and A. Zaslavsky, Phys. Rev. B 64, 85319 (2001).

[3] L. Saminadayar, D. C. Glattli, Y. Jin, B. Etienne, Phys. Rev. Lett. 79, 2526 (1997). R. de-Piccitio, M. Reznikov, M. Heiblum, V. Umansky, G. Bunin and D. Mahalu, Nature 389, 162, (1997).

[4] F.D.M. Haldane, Phys. Rev. Lett., 45, 1358, (1980); ibid., J. Phys. C14, 2585, (1981).

[5] I. Safi and H.J. Shulz, ibid., R17040 (1995). See also I. Safi and H.J. Shulz, in Quantum Transport in Semiconductor Submicron Structures, ed. by B. Kramer (Kluwer Academic Press, Dordrecht, 1995);

[6] D.L. Maslov and M. Stone, Phys Rev. Lett. B52, R5539 (1995).

[7] V.V. Ponomarenko, Phys Rev. Lett. B52, R8666 (1995). 
[8] W. Apel and T.M. Rice, Phys Rev. B 26, 7063 (1982).

[9] S. Tarucha, T. Honda and T. Saku, Solid State Commun. 94, 413 (1995).

[10] X.G. Wen, Phys. Rev. B 41, 12838 (1990); Adv. Phys. 44, 405 (1995)

[11] D.C. Tsui, H.L. Stormer and A.C. Gossard, Phys Rev. Lett. 48, 1559 (1982).

[12] A. Yacoby, H.L. Stormer, N.S. Wingreen, L.N. Pfeiffer, K.W. Baldwin and K.W. West, Phys. Rev. Lett. 77, 4612 (1996).

[13] R. de Picciotto, H.L. Stormer, A. Yacoby, L.N. Pfeiffer, K.W. Baldwin and K.W. West, Phys. Rev. Lett. 851730 (2000).

[14] R. de Picciotto, H.L. Stormer, L.N. Pfeiffer, K.W. Baldwin and K.W. West, Nature 41151 (2001).

[15] M. Bockrath, D.H. Cobden, J. Lu, A.R. Rinzler, R.E. Smalley, L. Balents and P.L. McEuen, Nature, 397, 598 (1999).

[16] J. Kong, E.Y. Yenilmez, T.W. Tombler, W. Kim, H. Dai, R.B. Laughlin, L. Liu, C.S. Jayanthi and S.Y. Wu, Phys Rev. Lett. 87, 106801 (2000).

[17] A.Yu. Kasumov, R. Deblock, M. Kociak, B. Reulet, H. Bouciat, I.I. Khodos, Yu.B. Gorbatov, V.T. Volkov, C. Journet and M. Burghard, Science, 284, 1508 (1999).

[18] R. Saito, G. Dresselhaus and M.S. Dresselhaus, Physical Properties of Carbon Nanotubes, Imperial College Press (1998).

[19] C. Dekker, Phys. Today, 52, No. 5, 22 (May 1999).

[20] S. Iijima, Nature, 354, 56 (1991).

[21] R. Egger, A. Bachtold, M. S. Fuhrer, M. Bockrath, D. H. Cobden, and P. L. McEuen, cond-mat/0008008.

[22] S. Frank, P. Poncharal, W.A. de Heer, Science, 280, 1744 (1998).

[23] R. Egger and A.O. Gogolin, Phys Rev. Lett. 79, 5082 (1997); C.L. Kane, L. Balents and M.P.A. Fisher, ibid., 5086 (1997).

[24] R. Landauer, Phil. Mag. 21, 863 (1970); M. Büttiker, Phys. Rev. B38 9375 (1988).

[25] C. Chamon and E. Fradkin, Phys. Rev. B56, 2012 (1997).

[26] In this work we shall consider only the above two boundary conditions as far as end contacts are concerned - A more general thermodynamic approach including a prediction on the shot-noise spectrum under a variety of boundary conditions will be discussed in K.-I. Imura, K.V. Pham, P. Lederer and F. Piéchon, in preparation.

[27] Of course, the stationary component of $\rho_{ \pm}^{(0)}(x, t)$ corresponds to the number of bare excitations, i.e., those at $+k_{\mathrm{F}}$ and at $-k_{\mathrm{F}}: Q_{ \pm}^{(0)}=\int_{-L / 2}^{L / 2} \rho_{ \pm}^{(0)}(x, t)$, where $Q_{ \pm}^{(0)}=$ $\frac{1}{2}(N \pm J) \cdot Q_{ \pm}^{(0)}$ and $Q_{ \pm}$are related through the matrix
$\boldsymbol{\Omega}:\left[\begin{array}{l}Q_{+} \\ Q_{-}\end{array}\right]=\boldsymbol{\Omega}\left[\begin{array}{l}Q_{+}^{(0)} \\ Q_{-}^{(0)}\end{array}\right]$, where $\boldsymbol{\Omega}=\frac{1}{2}\left[\begin{array}{cc}1+g & 1-g \\ 1-g & 1+g\end{array}\right]$.

[28] A similar calculation for the ground state can be found in A.Yu. Alekseev, V.V. Cheianov and J. Froehlich, Phys. Rev. B54 R17320 (1996).

[29] A.Yu. Alekseev, V.V. Cheianov and J. Froehlich, condmat/9706061; Phys. Rev. Lett. 81, 3503 (1998).

[30] I. Safi, Eur. Phys. J. B12, 451 (1999).

[31] R. Egger and H. Grabert, Phys. Rev. Lett. 79, 3463 (1997); R. Egger and H. Grabert, Phys. Rev. B58, 10761 (1998).

[32] C.L. Kane and M.P.A. Fisher, Phys. Rev. Lett. 68, 1220 (1992).

[33] In this case the crossover from weak tunneling to ohmic regime would be worth mentioning 25. The idea is to map the system to an equivalent quasiparticle $\leftrightarrow$ electron tunneling duality model 32,42] at effective filling factor $\nu_{\text {eff }}$. The system (=sample+reservoirs) is first modelized as a chiral electron tunneling model with different fictitious filling factors, i.e., $\nu_{1}=g$ and $\nu_{2}=1$. Then a rotation in the space of bosonic field is applied. $\nu_{\text {eff }}$ is obtained by comparing the scaling dimension of electron tunneling operators in the original and rotated models: $\frac{1}{\nu_{1}}+\frac{1}{\nu_{2}}=\frac{2}{\nu_{\text {eff }}}$. In the strong electron tunneling (ohmic) limit we have a boundary condition $I=\nu_{\text {eff }} \frac{e^{2}}{h}\left(V_{S}-V_{-}\right)$, $I=\nu_{\text {eff }} \frac{e^{2}}{h}\left(V_{+}-V_{D}\right)$. Together with a voltage drop equation: $I=\nu \frac{e^{2}}{h}\left(V_{+}-V_{-}\right)$this amounts to the boundary condition (3).

[34] A. Kawabata, J. Phys. Soc. Japan 65, 30 (1996).

[35] Y. Oreg and A.M. Finkel'stein, Phys. Rev. B54, R14265 (1996).

[36] M.P.A. Fisher and L.I. Glazman, in Mesoscopic Electron Transport, edited by L.L. Sohn, L.P. Kouwenhouven and G. Schoen, NATO series E, Vol. 345, 331 (Kluwer Academic Publishing, Dordrecht, 1997).

[37] B. Trauzettel, R. Egger and H. Grabert, condmat/0109022.

[38] C. Bena, S. Vishveshwara, L. Balents, and M.P.A. Fisher, cond-mat/0008188.

[39] V.V. Ponomarenko and N. Nagaosa, Solid State Commun. 110, 321, (1999).

[40] For $N_{R}\left(N_{L}\right)$ point-like contacts connected to the right (left) reservoir, the two-terminal conductance is given in the strong-coupling for each contact as

$$
G_{\mathrm{SD}}=\nu \frac{e^{2}}{h} \frac{\left[1-\left(-\frac{1-\nu}{1+\nu}\right)^{N_{L}}\right]\left[1-\left(-\frac{1-\nu}{1+\nu}\right)^{N_{R}}\right]}{1-\left(-\frac{1-\nu}{1+\nu}\right)^{N_{L}+N_{R}}} .
$$

[41] A. Furusaki and N. Nagaosa, Phys. Rev. B47, 4631 (1993).

[42] A. Schmid, Phys. Rev. Lett. 51, 1506 (1983). 